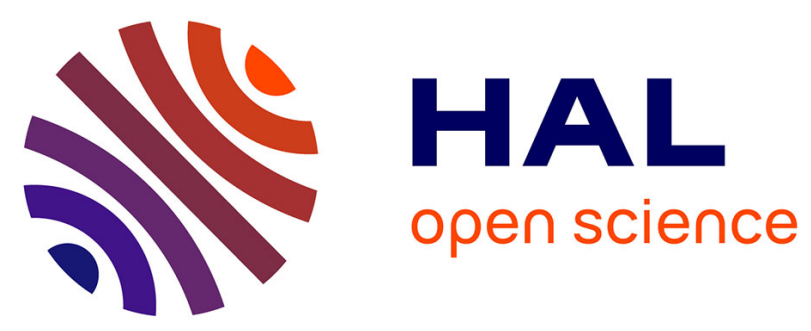

\title{
FMT signal options and associated receiver architectures for GNSS
}

\author{
Axel Javier Garcia Peña, Olivier Julien, Christophe Macabiau, Jong-Hoon \\ Won, Diana Fontanella, Matteo Paonni, Bernd Eissfeller, Andrea Emmanuele, \\ Marco Luise, Francesca Zanier, et al.
}

\section{To cite this version:}

Axel Javier Garcia Peña, Olivier Julien, Christophe Macabiau, Jong-Hoon Won, Diana Fontanella, et al.. FMT signal options and associated receiver architectures for GNSS. IEEE/ION PLANS 2012, Position Location and Navigation Symposium, Apr 2012, Myrtle Beach, United States. pp 898-912, 10.1109/PLANS.2012.6236833 . hal-01069623

\section{HAL Id: hal-01069623 \\ https://hal-enac.archives-ouvertes.fr/hal-01069623}

Submitted on 29 Sep 2014

HAL is a multi-disciplinary open access archive for the deposit and dissemination of scientific research documents, whether they are published or not. The documents may come from teaching and research institutions in France or abroad, or from public or private research centers.
L'archive ouverte pluridisciplinaire HAL, est destinée au dépôt et à la diffusion de documents scientifiques de niveau recherche, publiés ou non, émanant des établissements d'enseignement et de recherche français ou étrangers, des laboratoires publics ou privés. 


\section{FMT Signal Options and Associated Receiver Architectures for GNSS}

\author{
A. Garcia-Pena, O. Julien, C. Macabiau \\ ENAC \\ Toulouse, France
}

A. Emmanuele, M. Luise

Department of Information Engineering

University of Pisa

Pisa, Italy

\author{
J-H. Won, D. Fontanella, M. Paonni, B. Eissfeller \\ The Institute of Space Technology and Space Application \\ University FAF Munich \\ Munich, Germany
}

\author{
F. Zanier, G. Lopez-Risueno \\ European Space Agency \\ ESTEC, The Netherlands
}

\begin{abstract}
Non classical signals, signals not made of rectangular shaped pulses, are being analyzed as possible candidates for the next generation of GNSS signals. One such a signal is the filtered multi-tone (FMT) which is a multi-carrier signal (MC) having a MC-DS-CDMA structure: each subcarrier transports a classical GNSS signal but without using rectangular shaped pulses. Some studies have been conducted on the FMT signal performances such as the out-of-band (OOB) emission and multipath resistance. However, due to its MC nature, the distribution of the transmitted data onto the different subcarriers becomes a relevant factor to take into account on the final signal design. In this paper, different data distributions are proposed in order to enhance different signal performance and the receiver structure associated to each data distribution is also analyzed.
\end{abstract}

Keywords-FMT signal, filtered multi-tone, data distribution, correlator block, demodulator block, acquisition, tracking, Doppler ambiguity

\section{INTRODUCTION}

The design of current and near future Signals-In-Space (SIS) of Global Navigation Satellite Systems (GNSSs) has traditionally been based on Direct Sequence-Spread Spectrum signals (DD-SS) where the shaping of the spreading PRN code sequence chips is a rectangular waveform and the modulation technique chosen to transmit the binary information data is a BPSK modulation.

The use of rectangular chip pulses was justified by several advantages in terms of simplicity of the generation scheme and of the receiver architecture: simple multiplexing schemes, constant envelope signals in order to use optimally the payload High Power Amplifier (HPA) at its saturation point, implementation of 1-bit quantization of the local replica signals in the receivers, etc [1]. However, rectangular pulse shaped signals present high out-of-band (OOB) emissions which are a major drawback since the final broadcasted signal must be strongly filtered and thus significantly deformed [2]. Therefore, new bandwidth efficient modulations are being inspected as possible candidates for future GNSS signals 0 .
One possible such candidate is the filtered multi-tone (FMT). A FMT signal is a multicarrier (MC) type of modulation using band limited pulses, is especially resistant to multipath and has very low OOB emissions. The principle of a MC modulation in the telecommunication field is to convert a serial high-rate data stream onto multiple parallel low-rate substreams; each of these sub-streams is transmitted on a specific carrier, referred to as sub-carrier. However, this definition has to be adapted to GNSS signals which in addition to carry information data also have to allow for the acquisition and tight synchronization with the incoming signal.

A possible solution is the transmission of traditional GNSS signals in each subcarrier, such as DS-SS BPSK signals with square-root raised cosine (SRRC) shaped bits [4]. However, there is one remaining part of this adjustment that has not been addressed in previous works: the most interesting distribution of the data information between the different subcarriers. This search for an optimal data distribution among the subcarriers has to be done in parallel with an investigation of the associated receiver and receiver performance (correlator structure, autocorrelation function, etc). This also means the acquisition, tracking and demodulation performance of the signal.

The aim of this paper is first to present and to analyze different data distributions on a FMT GNSS signal. Each distribution is designed in order to enhance different properties of the signal such as the time and frequency diversity of the data, and the optimization of the acquisition and tracking performance. Second, this paper aims at presenting suitable correlator structures for each of the distributions considered in order to allow for the acquisition and tracking of each of them. Preliminary performance assessment in terms of acquisition, tracking and data demodulation should aid the decision of future FMT GNSS signal designs.

This paper is structured in 9 different sections including this introduction. First, a mathematical description of a FMT 
signal is given. Second, the description and advantages of different data distributions are given. Third, correlator block structures for each different data distribution are described and analyzed, in particular, a discussion on the opportunity to use FFT-based correlators. Fourth, the acquisition block, strategies and preliminary performances are addressed. Fifth, the tracking architecture and performance is given. Sixth, demodulator architectures for each data distribution are given. Seventh, a summary is provided and finally the conclusions are presented.

\section{FMT SignAl MATHEMATICAL MODEL}

The equivalent baseband mathematical model of a received FMT signal at the RF front-end block is presented first [4]. Second, the pass-band mathematical model is given. Third, the numerical values of the FMT signal parameters are provided.

A. Equivalent baseband mathematical model of a FMT signal

$$
\begin{gathered}
x(t)=\sum_{k=-\infty}^{+\infty} d_{k}\left(t-k \cdot X \cdot T_{R}, t\right) \\
d_{k}\left(t^{\prime}, t\right)=\sum_{n \in D(M)} A_{n} \cdot d_{n}^{k} \cdot c_{F(n)}\left(t^{\prime}\right) \cdot e^{j 2 \pi f_{n} t} \\
c_{F(n)}(t)=\sum_{m=0}^{G-1} c_{\bmod (m+F(n), G)} \cdot g S R R C_{T s}^{\text {mono }}\left(t-m \cdot T_{s}\right)
\end{gathered}
$$

- $\mathrm{T}_{\mathrm{R}}: \mathrm{PRN}$ code period

- $\quad \mathrm{X}$ : Number of PRN code periods spanning a data symbol

- $\mathrm{A}_{\mathrm{n}}$ : Amplitude of the subcarrier $n$ with $\mathrm{A}_{\mathrm{n}}=\mathrm{A}_{-\mathrm{n}}$.

- $\mathrm{d}_{\mathrm{n}}{ }^{\mathrm{k}}$ : Data symbol transmitted at epoch $k$ at the subcarrier $n$. Bounded between $\left(0, \mathrm{X} \cdot \mathrm{T}_{\mathrm{R}}\right]$

- $\quad$ M: Number of active subcarriers

- $\mathrm{D}(\mathrm{M})$ : Symmetric ensemble of $\mathrm{M}$ integers: $n \in$ $D(M) \leftrightarrow-n \in D(M)$

- G: Number of chips of a PRN code

- $\mathrm{g}_{\mathrm{SRRC}}{ }^{\text {mono }} \mathrm{x}(\mathrm{t})$ : Monomodal square-root raised cosine pulse (SRRC) of duration $\mathrm{X}$

- $\mathrm{T}_{\mathrm{s}}$ : FMT symbol duration. In this paper, $\mathrm{T}_{\mathrm{s}}$ is equal to the PRN code chip duration of a subcarrier

- $\quad \mathrm{c}_{\mathrm{x}}$ : Chip $x$ of the PRN code

- $\operatorname{Mod}(\mathrm{x}, \mathrm{y})$ : Mathematical operation of $x$ modulo $y$

- $F(n)$ : Function providing an integer between [0,G-1]

- $\mathrm{f}_{\mathrm{n}}$ : Frequency of subcarrier $n$.

In order to simplify the mathematical model, subcarriers are conceived in symmetric pairs with respect to the $0 \mathrm{~Hz}$ axis:

$$
f_{-n}=-f_{n}
$$

The exact subcarrier frequency configuration is:

$$
f_{n}=n \cdot \Delta f
$$

- $\Delta \mathrm{f}$ : Spectral difference between subcarriers.

Function $\mathrm{F}(\mathrm{n})$ is responsible of avoiding the inherent Doppler ambiguity effect of a FMT signal. More information about $\mathrm{F}(\mathrm{n})$ and this phenomenon is provided in sections V.E and V.F but for now note that due to this function, each subcarrier implements the same PRN code sequence but with a different circular cyclic shift. From equation (5), it is observed that $\Delta \mathrm{f}$ is equal to the bandwidth of the subcarriers. Hence, it is equal to the SRRC pulse bandwidth:

$$
\Delta f=B W_{\text {sub }}=\frac{1+\alpha}{T_{s}}
$$

- $\alpha$ : SRRC pulse roll-off factor

Another expression for the equivalent baseband mathematicalmodel of a received FMT signal is given below:

$$
x(t)=\sum_{n \in D(M)} A_{n} \cdot\left(\sum_{k=-\infty}^{+\infty} d_{n}^{k} \cdot c_{F(n)}\left(t-k \cdot X \cdot T_{R}\right)\right) \cdot e^{j 2 \pi f_{n} t}
$$

Equation (7) shows that a FMT signal is simply the parallel broadcasting of several traditional GNSS signals in different subcarriers (each one with a different signal carrier central frequency). The other difference is the PRN code chips shape: a SRRC pulse.

Finally, since a FMT signal is a MC signal, the chip time of the total FMT signal, $\mathrm{T}_{\mathrm{c}}$, is defined as:

$$
T_{c}=T_{S} / N
$$

- $\quad \mathrm{N}$ : Number of subcarriers

B. Equivalent passband mathematical model of a FMT signal

$$
\begin{gathered}
x(t)=\sum_{n \in D(M)} A_{n} \cdot\left(\sum_{k=-\infty}^{+\infty} d_{n}^{k} \cdot c_{F(n)}\left(t-k \cdot X \cdot T_{R}\right)\right) \\
\cdot\left(\cos \left(2 \pi f_{n} t\right) \cdot \cos \left(2 \pi f_{c} t\right)-\sin \left(2 \pi f_{n} t\right) \cdot \sin \left(2 \pi f_{c} t\right)\right)
\end{gathered}
$$

- $\mathrm{f}_{\mathrm{c}}$ : Signal carrier frequency

Equation (9) shows that the transmitted signal has in-phase and quadrature-phase components.

\section{Numerical Values}

This analysis was conducted in the framework of the ADVISE project. Therefore, the numerical values of this project are used in the entire paper for numerical results and figures with a slight variation in order to facilitate the different data distributions comparison [4].

- $\alpha=0.2$

- $\mathrm{T}_{\mathrm{c}}=1 / 13.55475 \mathrm{e} 6$ seconds

- $\mathrm{M}=60 ; \mathrm{N}=67$;

- $\mathrm{D}(\mathrm{M})=\{-34:-5 ; 5: 34\}$ and with $n \in D(M)$

The amplitude is calculated following the next equations:

$$
\begin{gathered}
A_{n}= \begin{cases}1-\frac{4 \cdot\left|n+k^{\prime}\right|}{M} & n<0 \\
1-\frac{4 \cdot\left|n-k^{\prime}\right|}{M} & n>0\end{cases} \\
k^{\prime}=\frac{N-1}{2}-\frac{M-2}{4}
\end{gathered}
$$




\section{DIFFERENT DATA DISTRIBUTIONS}

Different distributions of the data carried in the section II FMT signal are described. Each different data distribution is designed in order to enhance specific characteristics of the FMT signal and thus, each distribution is oriented to a different kind of application: only geo-ranging, only data communication or geo-ranging and data communication.

The different distributions are graphically described in order to help the reader's comprehension. The horizontal axis represents the time domain, where each big rectangular box surrounded by white color represents the whole FMT signal inside a PRN code period. The vertical axis represents the frequency domain where each small rectangular box separated from the others by black lines represents a subcarrier (a subcarrier during a PRN code period). Finally, the color of each box represents the symbol carried by that the specific subcarrier during a PRN code period of time.

Some important definitions and characteristics must be given before presenting the different data distributions. The group symbol is defined as the group of parallel transmitted symbols which start their transmission at the same instant $t_{1}$ and end their transmission at the same instant $t_{2}$. The different data distributions are designed in order to transmit a continuous flow of group symbols. The group symbol length is the time between $[\mathrm{t} 2, \mathrm{t} 1]$, and the group symbol structure is the time-frequency distribution of symbols making the group symbol. Finally, in order to set a fair comparison between the different data distributions, any data symbol transmitted in any data distribution always has the same energy: the number of boxes representing the data is always the same.

\section{A. First Distribution: Pilot component}

Figure 1 presents the $1^{\text {st }}$ data distribution which is designed exclusively for a pilot component since the carried data is the same for all the time-frequency symbols (or boxes). The group symbol is reduced to an infinite 1 symbol.

Figure 1 shows that this distribution presents the maximum possible degree of frequency and time diversity.

\section{B. Second Distribution: Quasi-Pilot or Data component}

Figure 2 presents the $2^{\text {nd }}$ data distribution which is designed for Quasi-Pilot components or for data components giving the same priority to the geo-ranging capability as to the data communication.

$2^{\text {nd }}$ data distribution group symbol is made of 1 data symbol and the group symbol length depends on the targeted application: from 1 PRN code period for a data component to several PRN code periods for a quasi-pilot component.

This data distribution presents the highest frequency diversity degree but the lowest time diversity degree when the data symbol only spans 1 PRN code (data component).

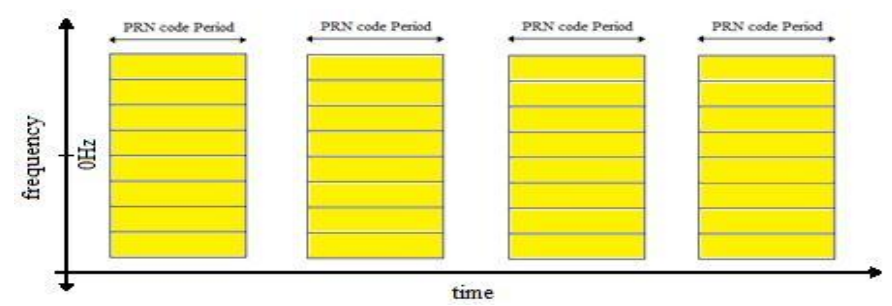

Figure 1: $1^{\text {st }}$ Data distribution - Pilot component

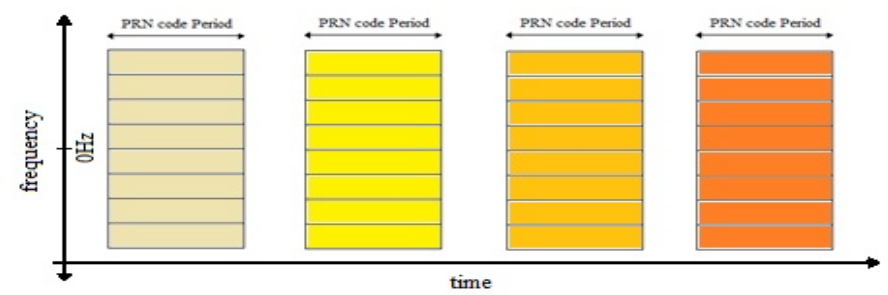

Figure 2: Example of $2^{\text {nd }}$ Data distribution - Quasi-Pilot or Data component

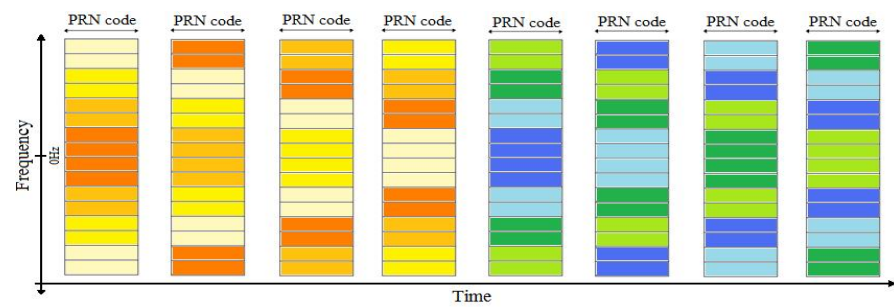

Figure 3: Example of $3^{\text {rd }}$ Data distribution - Data component with geo-ranging capabilities

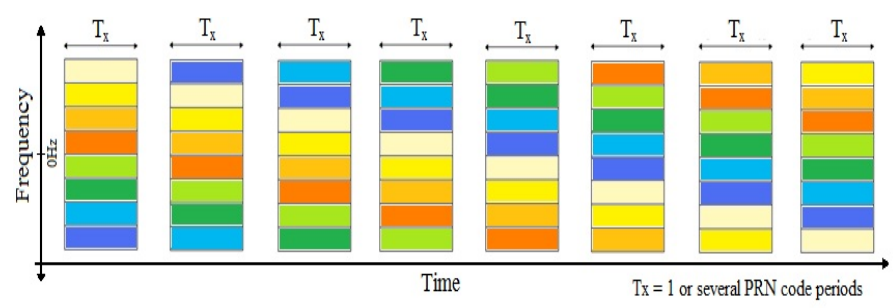

Figure 4: Example of $4^{\text {th }}$ Data distribution - data component without geo-ranging capabilities

\section{Third Distribution: Data component with geo-ranging}

Figure 3 presents the $3^{\text {rd }}$ data distribution which is designed for data components giving priority to the data communication over the geo-ranging capabilities.

The group symbol is made of 2 or more data symbols until a maximum number equal to half the number of active subcarriers (M).

The group symbol length is equal to the number of $2^{\text {nd }}$ data distribution group symbol length multiplied by the number of parallel transmitted symbols (U). The group symbol structure of consecutive transmitted group symbols is always the same.

Figure 3 shows a better time diversity for the $3^{\text {rd }}$ data distribution as for the $2^{\text {nd }}$ data distribution since the data symbol transmission is expanded in time. For example, if 1 data symbol spans only 1 PRN in the $2^{\text {nd }}$ data distribution, in the $3^{\text {rd }}$ data distribution this data symbol spans 2 or more PRN 
codes (4 in the example of Figure 3). Moreover, both data distributions have the same frequency diversity since a data symbol is broadcasted by all the subcarriers.

Therefore, the $3^{\text {rd }}$ data distribution should have better demodulation performance than the $2^{\text {nd }}$ data distribution due to the higher time diversity. However, its acquisition performance is worse (see section V) but they have the same tracking performance (see section 0 ).

Three more remarks can be made about the $3^{\text {rd }}$ data distribution. First, symmetric subcarriers with respect to the 0 $\mathrm{Hz}$ frequency axis must carry the same data symbol in order to allow geo-ranging capabilities (see section IV.C). Second, the number of adjacent subcarriers carrying the same data symbol at the same PRN code transmission determines the acquisition performance (see section V.B). Third, the signal data rate is maintained constant.

\section{Fourth Distribution: data component without geo-ranging}

Figure 4 shows the $4^{\text {th }}$ data distribution which is designed for data components not providing geo-ranging capabilities. The $4^{\text {th }}$ data distribution group symbol is made of a number of data symbols equal to the number of active subcarriers. The group symbol length is called $\mathrm{T}_{\mathrm{D}}$ (data symbol duration) which depends on the signal design, from 1 PRN code period to several PRN code periods (the addition of all the Tx must be equal to $T_{D}$ ). The group symbol structure must be the same from 1 group symbol to next broadcasted one in order to allow the correct group symbol demodulation.

This data distribution presents the highest time diversity degree as well as a large frequency diversity degree. In fact, the frequency diversity depends on $T_{x}$ where $T x$ has to be a multiple of one PRN code period. If $T_{x}$ is shorter than one PRN code the data symbol demodulation is degraded as presented in section VII.C.

\section{CORRELATOR BLOCK STRUCTURES}

In this section, the $1^{\text {st }}, 2^{\text {nd }}$ and $3^{\text {rd }}$ data distributions correlator blocks are described, their output mathematical expressions in presence of thermal noise are given and the correlation functions are drawn. The mathematical expressions as well as the correlations figures are given for non-coherent outputs for the following reasons. $2^{\text {nd }}$ and $3^{\text {rd }}$ data distributions are designed to be used as data communication links but also have to provide geo-ranging capabilities. In that latter case, the data component is combined with the pilot component. However, in order to obtain a constructive combination of both components, we need either to know the broadcasted data or to use non-coherent correlator outputs. If the broadcasted data is known, all the data distributions become exactly as the $1^{\text {st }}$ distribution from the receiver point of view and there is no interest in comparing them. Besides, this assumption is not valid for the acquisition process. Therefore, in this paper, we are mainly interested in the non-coherent outputs of the $2^{\text {nd }}$ and $3^{\text {rd }}$ data distribution correlator outputs.

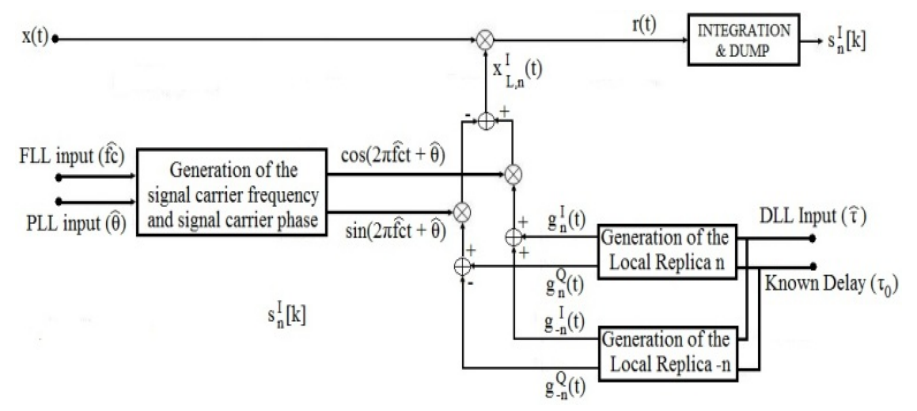

Figure 5: Correlator block scheme of 2 symmetric subcarriers

- $\quad x(t)$ : Baseband FMT received signal at the RF output

- $\quad x_{L, n}^{I}(t)$ : In-phase generated local replica of the $n$ and -n actives subcarriers

- $\quad \mathrm{s}_{\mathrm{n}}^{\mathrm{I}}[\mathrm{k}]$ : Correlator output of the in-phase generated local replica of symmetric subcarriers $f_{n}$ and $f_{-n}$ at epoch $\mathrm{k}$

- $\quad \mathrm{g}^{\mathrm{Y}}{ }_{\mathrm{i}}(\mathrm{t})$ : In-phase or quadrature-phase (depends on $\mathrm{Y}$ ) generated local replica of the $\mathrm{i}^{\text {th }}$ active subcarrier

- $\widehat{f}_{c}$ : Estimated signal carrier frequency by the FLL

- $\hat{\theta}$ : Estimated signal carrier phase by the PLL

- $\hat{\tau}$ : Estimated signal code delay by the DLL

- $\tau_{0}$ : Known delay (early or late correlator blocks)

The $4^{\text {th }}$ data distribution correlator block is not given in this section since it cannot provide geo-ranging capabilities.

Finally, in this section, a discussion on the opportunity to use FFT-based correlators instead of the current correlator structures presented in this paper is provided in order to justify the paper's choice.

\section{A. Correlator block of 2 symmetric subcarriers}

The $1^{\text {st }}, 2^{\text {nd }}$ and $3^{\text {rd }}$ data distributions have a group symbol structure where pairs of symmetric subcarriers with respect to the $0 \mathrm{~Hz}$ frequency axis carry the same data symbol. Therefore, we can design their correlator blocks as different combinations in frequency and in time of fundamental correlator blocks of 2 symmetric subcarriers. Figure 5 presents a scheme of the correlator block of 2 symmetric subcarriers.

$$
\begin{aligned}
x_{L, n}^{I}(t)= & \left(g_{n}^{I}(t)+g_{-n}^{I}(t)\right) \cos \left(2 \pi \widehat{f}_{c} t+\hat{\theta}\right) \\
& \quad\left(g_{n}^{Q}(t)-g_{-n}^{Q}(t)\right) \sin \left(2 \pi \widehat{f}_{c} t+\hat{\theta}\right) \\
g_{n}^{I}(t)= & A_{n} \cdot c_{F(n)}\left(t-\hat{\tau}-\tau_{0}\right) \cdot \cos \left[2 \pi f_{n}\left(t-\hat{\tau}-\tau_{0}\right)\right] \\
g_{n}^{Q}(t)= & A_{n} \cdot c_{F(n)}\left(t-\hat{\tau}-\tau_{0}\right) \cdot \sin \left[2 \pi f_{n}\left(t-\hat{\tau}-\tau_{0}\right)\right]
\end{aligned}
$$

Figure 5 and equations (13) and (14) show that the local replicas of subcarriers $f_{n}$ and $f_{-n}$ are already generated in its subcarrier frequency. This means that in addition to generating the SRRC shaped PRN code, the cosine and sine translating the PRN code into its corresponding frequency are also generated in the local replica block. Therefore, as expressed in equations (13) and (14), the instantaneous phase of $f_{n}$ and $f_{-n}$ local replicas depend on the estimated signal code delay $(\hat{\tau})$ and the known delay $\left(\tau_{0}\right)$. 
The quadrature-phase local replica of the combined $f_{n}$ and $f_{-n}$ subcarriers is obtained similarly to the in-phase local replica:

$$
\begin{aligned}
x_{L, n}^{Q}(t)=-\left(g_{n}^{I}(t)+\right. & \left.g_{-n}^{I}(t)\right) \cos \left(2 \pi \widehat{f}_{c}+\hat{\theta}\right) \\
& -\left(g_{n}^{Q}(t)-g_{-n}^{Q}(t)\right) \sin \left(2 \pi \widehat{f}_{c}+\hat{\theta}\right)
\end{aligned}
$$

The mathematical expression of the correlator output depicted in Figure 5 is presented next. For simplifications purposes and without loss of generality, the number of PRN code periods transporting the same data symbol is set to 1 $(\mathrm{X}=1)$. Another important simplification of the integration time is that the part of the received signal which is not contained inside $(\mathrm{k}-1 / 2) \mathrm{T}_{\mathrm{R}}$ and $(\mathrm{k}+1 / 2) \cdot \mathrm{T}_{\mathrm{R}}$ has been removed from the equation.

$$
s_{n}^{I}[k]=\frac{1}{T_{R}} \int_{(k-1 / 2) T_{R}}^{(k+1 / 2) T_{R}} x(t) \cdot x_{L}^{I}(t) d t
$$

Mathematical expression of $\mathrm{s}_{\mathrm{n}}^{\mathrm{I}}[\mathrm{k}]$ can be approximated as:

$$
\begin{gathered}
s_{n}^{I}[k] \approx \\
\frac{A_{n}^{2}}{2} \cdot\left(d_{n}^{k} \cdot R_{F(n)}(\Delta \tau)+d_{-n}^{k} \cdot R_{F(-n)}(\Delta \tau)\right) \cdot \cos \left(2 \pi f_{n} \Delta \tau\right) \cdot G(k, 0) \\
-\frac{A_{n}^{2}}{2} \cdot\left(d_{n}^{k} \cdot R_{F(n)}(\Delta \tau)-d_{-n}^{k} \cdot R_{F(-n)}(\Delta \tau)\right) \cdot \sin \left(2 \pi f_{n} \Delta \tau\right) \cdot G(k, \pi / 2) \\
G(k, \alpha)=\int_{(k-1 / 2) T_{R}}^{(k+1 / 2) T_{R}} \cos (2 \pi \Delta f t+\Delta \theta+\alpha) d t
\end{gathered}
$$

- $\Delta f=f c-\widehat{f c}$, signal carrier frequency estimation error

- $\Delta \theta=\theta-\hat{\theta}$, signal carrier phase estimation error

- $\Delta \tau=\tau-\hat{\tau}$, signal code delay estimation error

- $R_{F(i)}(x)$ : Correlation function evaluated at $\mathrm{x}$ of the circularly shifted PRN code of subcarrier $f_{i}$. The PRN code was F(i) chips circularly shifted.

- $d_{i}^{k}$ : Data symbol carried by subcarrier i at epoch $\mathrm{k}$.

Equation (17) has been approximated by following two steps. First, terms at $2 \mathrm{f}_{\mathrm{c}}$ and at $2 \mathrm{f}_{\mathrm{n}}$ are removed by the integration block. Second, the total integration is approximated by the multiplication of two integrations: the integration of the PRN code and the integration of the sine and cosine terms at $\Delta \mathrm{f}$ ( $\mathrm{G}$ function) [5]. This approximation of the integration is possible since each individual subcarrier is a SRRC shaped PRN code signal with a multiplicative constant, either $\cos \left(2 \pi f_{n} \Delta \tau\right)$ or $\sin \left(2 \pi f_{n} \Delta \tau\right)$. Simulations not presented in this paper were run to verify this hypothesis.

Expression (18) can be further simplified since $R_{F(n)}$ correlation function is equal to $R_{F(-n)}$ (the same two PRN codes but circularly shifted) and due to the imposed symmetric subcarrier with respect to the $0 \mathrm{~Hz}$ frequency condition: $d_{i}^{k}=d_{-i}^{k}$. Therefore expression (18) is equal to:

$$
\begin{aligned}
s_{n}^{I}[k] \approx A_{n}^{2} \cdot d_{n}^{k} \cdot R_{c c}(\Delta \tau) \cdot \cos \left(2 \pi f_{n} \Delta \tau\right) & \cdot \operatorname{sinc}\left(\pi \Delta f T_{R}\right) \cdot \cos \left(\pi \Delta f 2 k T_{R}+\Delta \theta\right)
\end{aligned}
$$

- $\quad \mathrm{R}_{\mathrm{cc}}(\mathrm{x})$ : PRN code autocorrelation function

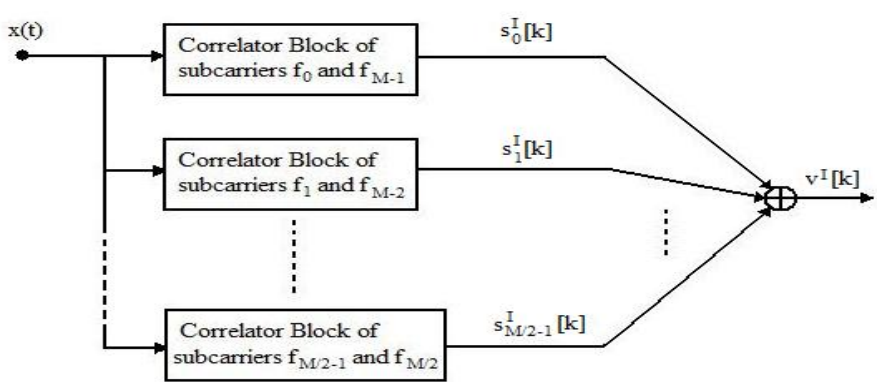

Figure 6: Correlator A block scheme

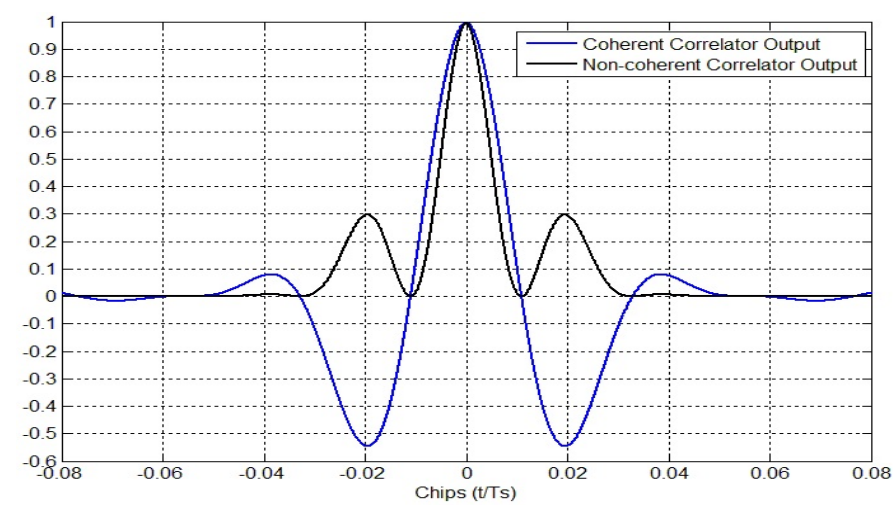

Figure 7: Normalized coherent and non-coherent correlator $A$ outputs

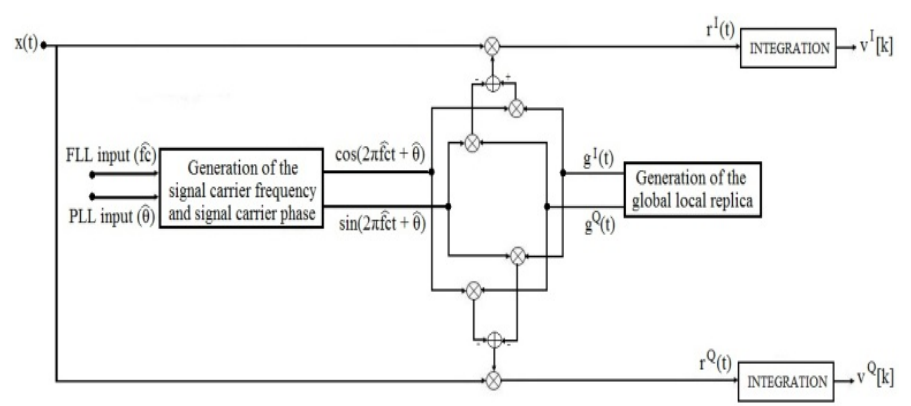

Figure 8: Final correlator A block scheme

Equivalently, the quadrature-phase output of the 2 symmetric subcarriers correlator, $\mathrm{s}_{\mathrm{n}}^{\mathrm{Q}}[\mathrm{k}]$, is approximated as:

$$
\begin{aligned}
s_{n}^{I}[k] \approx A_{n}^{2} \cdot d_{n}^{k} \cdot R_{c c}(\Delta \tau) \cdot \cos \left(2 \pi f_{n} \Delta \tau\right) & \cdot \operatorname{sinc}\left(\pi \Delta f T_{R}\right) \cdot \sin \left(\pi \Delta f 2 k T_{R}+\Delta \theta\right)
\end{aligned}
$$

Finally, it can be seen that adding the squared expressions of (19) and (20), the signal carrier phase estimation error is removed and thus, the signal can be acquired and noncoherently tracked.

\section{B. $1^{\text {st }}$ and $2^{\text {nd }}$ data distributions correlator blocks}

In this section, the $1^{\text {st }}$ and $2^{\text {nd }}$ data distribution correlator blocks architecture is presented. This correlator is referred as "correlator A" from now on. The correaltor A architecture consists in coherently adding the outputs of the $M / 2$ symmetric subcarriers pairs correlator. This coherent addition is possible since all the subcarriers transport the same data symbol. Figure 6 shows a scheme of the in-phase correlator block output. 
The mathematical expressions of in-phase and quadraturephase correlator A outputs are:

$$
\begin{aligned}
v^{I}[k] \approx \operatorname{sinc}\left(\pi \Delta f T_{R}\right) & \cdot \cos \left(\pi \Delta f 2 k T_{R}+\Delta \theta\right) \cdot R_{c c}(\Delta \tau) \cdot d_{n}^{k} \\
& \cdot \sum_{n=1}^{M / 2} A_{n}^{2} \cdot \cos \left(2 \pi f_{n} \Delta \tau\right) \\
v^{Q}[k] \approx \operatorname{sinc}\left(\pi \Delta f T_{R}\right) & \cdot \sin \left(\pi \Delta f 2 k T_{R}+\Delta \theta\right) \cdot R_{c c}(\Delta \tau) \cdot d_{n}^{k} \\
& \cdot \sum_{n=1}^{M / 2} A_{n}^{2} \cdot \cos \left(2 \pi f_{n} \Delta \tau\right)
\end{aligned}
$$

Figure 7 shows the normalized coherent output of the correlator $\mathrm{A}, \mathrm{v}^{\mathrm{I}}[\mathrm{k}]$, when assuming a perfect signal carrier frequency estimation $(\Delta \mathrm{f} \approx 0)$ and a perfect signal carrier phase estimation $(\Delta \theta \approx 0)$. Figure 7 also shows the normalized noncoherent output of the correlator $\mathrm{A}$, the addition of the squared expressions of $\mathrm{v}^{\mathrm{I}}[\mathrm{k}]$ and of $\mathrm{v}^{\mathrm{Q}}[\mathrm{k}]$, when assuming perfect signal carrier frequency estimation $(\Delta \mathrm{f} \approx 0)$. The numerical values used to represent Figure 7 are given in section II.C.

One important remark that can be made from Figure 7 is that due to the coherent addition of all the 2 symmetric subcarrier correlator outputs, the final correlation of the FMT signal uses all the original bandwidth $(\mathrm{M} \cdot \Delta \mathrm{f})$ instead of the individual subcarrier bandwidth $(\Delta \mathrm{f})$. This observation is made on the $\mathrm{X}$ axis, which is normalized by $\mathrm{T}_{\mathrm{s}}$ (individual subcarrier PRN code chip time), where it can be observed that the main lobe width is much narrower than $2 \mathrm{~T}_{\mathrm{s}}\left(T_{s}=(1+\alpha) / \Delta f\right)$.

Figure 6 correlator block can be simplified because the cross-products between different subcarriers are 0 due to the orthogonality in frequency and the orthogonality between PRN codes: different circular shifts of the PRN code for each subcarrier and the PRN autocorrelation function is approximated as 0 outside the [-Ts, Ts]:

$$
\int_{-T_{R} / 2}^{T_{R} / 2} A_{n} \mathrm{c}_{\mathrm{F}(\mathrm{n})}(\mathrm{t}) \cdot \cos \left(2 \pi f_{n} t\right) \cdot A_{i} \mathrm{c}_{\mathrm{F}(\mathrm{i})}(t) \cdot \cos \left(2 \pi f_{i} t\right) d t=
$$

Figure 8 depicts the final correlator A block scheme which is exactly the same correlator as presented in 0 and [4].

\section{C. $3^{\text {rd }}$ data distribution correlator blocks $-1^{\text {st }}$ Option}

In this section, the first option of the correlator block scheme for the $3^{\text {rd }}$ data distribution is presented. This correlator is referred as "correlator B" from now on and only provides non-coherent outputs (data symbols removal). Remember that coherent outputs can only be used when the broadcasted data symbol values are known.

In correlator $\mathrm{B}$, the 2 symmetric subcarriers correlator block outputs are fed to a block called "smart addition block" which simply adds the outputs of the subcarriers transporting the same data symbol. Then, the outputs of the smart addition block are squared and linearly added in order to generate a new correlation function which also uses all the available bandwidth of the FMT signal $(\mathrm{M} \cdot \Delta \mathrm{f})$. Figure 9 shows the general scheme of the in-phase correlator block.

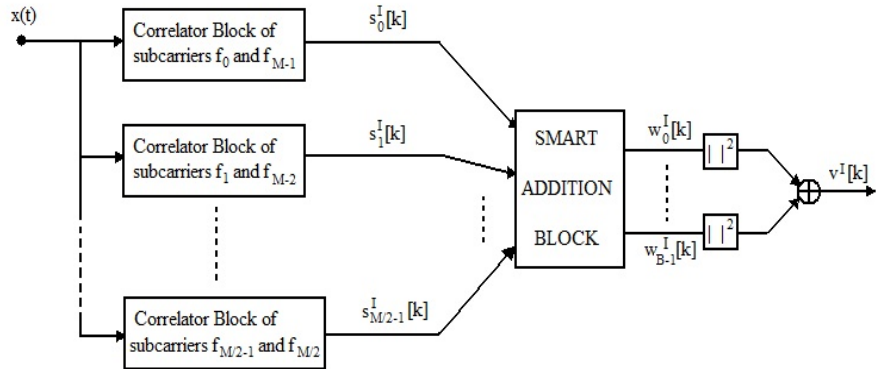

Figure 9: Correlator B block scheme

- $\quad$ B: number of parallel transmitted symbols by a group symbol

- $\quad \mathrm{w}_{b}^{\mathrm{I}}[\mathrm{k}]$ represents the addition of $\mathrm{s}_{\mathrm{n}}{ }^{\mathrm{I}}[\mathrm{k}]$ outputs carrying the same data symbol $\mathrm{d}_{\mathrm{k}}^{\mathrm{b}}$ at epoch $\mathrm{k}$.

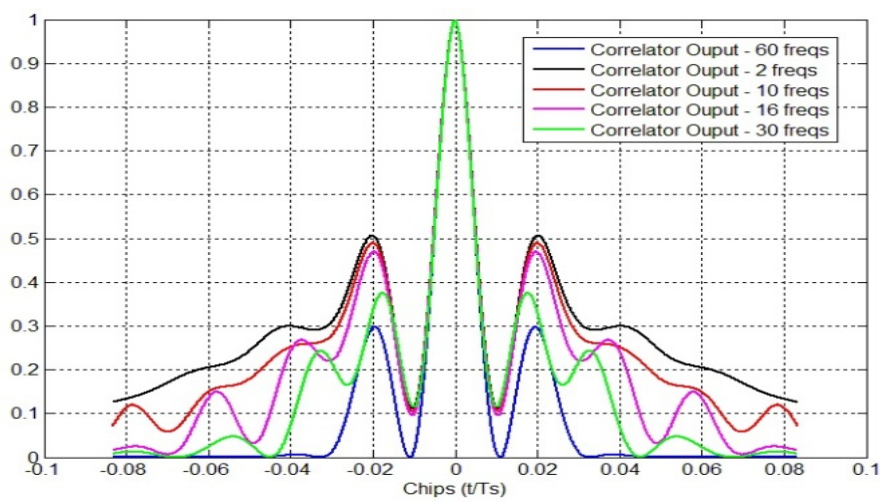

Figure 10: Normalized non-coherent correlator B output

TABLE I. NON-COHERENT OUTPUT MAIN LOBE TO SECONDARY LOBE RATIO FOR DIFFERENT SYMBOL GROUP CONFIGURATIONS OF THE CORRELATOR B BLOCK

\begin{tabular}{|c|c|}
\hline $\begin{array}{c}\text { Number of subcarriers carrying } \\
\text { the same symbol (T) }\end{array}$ & $\begin{array}{c}\text { Ratio between the main and } \\
\text { secondary lobes (dB) }\end{array}$ \\
\hline 2 & 2.95 \\
\hline 10 & 3.10 \\
\hline 16 & 3.28 \\
\hline 30 & 4.24 \\
\hline 60 & 5.26 \\
\hline
\end{tabular}

In order to use the correlator B without knowing at which instant of time the group symbol begins, it is necessary that the group symbol structure has an internal X PRN periodic structure being $X$ is the number of PRN code periods that a subcarrier transports the same symbol: subcarriers carrying the same symbol $\mathrm{y}_{0}$ at epoch $\mathrm{k}$ also carry the same symbol $\mathrm{y}_{1}$ at epoch $\mathrm{k}+1$. This is the case of the $3^{\text {rd }}$ data distribution because.

The number of subcarriers transporting the same data symbol is defined as (for simplifications purposes this number is assumed to be constant for all B symbols):

$$
T=M / B
$$

The mathematical expression of $\mathrm{w}_{\mathrm{b}}[\mathrm{k}]$ assuming $\mathrm{X}=1$ is:

$$
\begin{gathered}
w_{b}^{I}[k] \approx d_{k}^{b} \cdot R_{c c}(\Delta \tau) \cdot \operatorname{sinc}\left(\pi \Delta f T_{R}\right) \cdot \cos \left(\pi \Delta f 2 k T_{R}+\Delta \theta\right) \\
\cdot \sum_{n \forall\left(d_{k}^{b}=d_{k}^{n}\right)} A_{\mathrm{n}}^{2} \cos \left(2 \pi f_{n} \Delta \tau\right)
\end{gathered}
$$


And the mathematical expression of $v^{\mathrm{I}}[\mathrm{k}]$ is:

$$
\begin{gathered}
v^{I}[k] \approx R_{c c}{ }^{2}(\Delta \tau) \cdot \operatorname{sinc}^{2}\left(\pi \Delta f T_{R}\right) \cdot \cos ^{2}\left(\pi \Delta f 2 k T_{R}+\Delta \theta\right) \\
\cdot \sum_{b=0}^{B-1}\left(\sum_{n \forall\left(d_{k}^{b}=d_{k}^{n}\right)} A_{\mathrm{n}}^{2} \cos \left(2 \pi f_{n} \Delta \tau\right)\right)^{2}
\end{gathered}
$$

The mathematical expression of $\mathrm{v}^{\mathrm{Q}}[\mathrm{k}]$ is the same but changing the squared cosine by a squared sine.

Figure 10 shows the correlator B normalized non-coherent output: $\mathrm{v}^{\mathrm{I}}[\mathrm{k}]$ and $\mathrm{v}^{\mathrm{Q}}[\mathrm{k}]$ expressions addition when assuming perfect signal carrier frequency estimation $(\Delta \mathrm{f} \approx 0)$. Correlator $\mathrm{B}$ output depends on the value of $\mathrm{T}$ and thus Figure 10 shows the correlator B output for different values of $\mathrm{T}$ following the $3^{\text {rd }}$ data distribution group symbol structure: adjacent subcarriers transport the same symbol. Note that the 60 subcarriers configuration output is obviously equivalent to correlator A output. Besides, the group symbol structure must guarantee the data symbol subcarrier symmetry with respect to the $0 \mathrm{~Hz}$ frequency axis. Numerical values used to represent Figure 10 are given in section II.C.

Figure 10 shows that regardless of the number of subcarriers transmitting the same symbol, $\mathrm{T}$, the correlation function main peak has always the same width. The main difference is found on the secondary lobes amplitude which decreases with the increase of $\mathrm{T}$. The ratio between the main lobe and the secondary lobe of different configurations is shown in TABLE I.

\section{D. $3^{\text {rd }}$ data distribution correlator blocks $-2^{\text {nd }}$ Option}

In this section, the $2^{\text {nd }}$ option of the correlator block scheme for the $3^{\text {rd }}$ data distribution is presented. This correlator is referred as "correlator C" from now on and it is a correlator B evolution which takes advantage of the data symbol transmissions along the time on different subcarriers.

Correlator $\mathrm{C}$ structure consists in coherently accumulating in time consecutive correlator B outputs. All these outputs are fed to a block called "smart accumulation block" which coherently adds all the outputs transporting the same data symbol. Obviously, the correlator $\mathrm{C}$ needs to know the group symbol structure and at which instant it begins its transmission. Figure 11 presents the in-phase correlator $\mathrm{C}$ general scheme.

Mathematical expression of the $u^{\text {th }}$ output of the smart accumulation block, $\mathrm{e}_{\mathrm{u}}^{\mathrm{I}}[\mathrm{r}]$, is:

$$
\begin{gathered}
e_{u}^{I}[r]=\sum_{\substack{l=0 \\
b \forall\left(d^{u}=d_{k+l}^{b}\right)}}^{L-1} w_{b}[k+l] \\
\sum_{\substack{l=0 \\
b \forall\left(d^{u}=d_{k+l}^{b}\right)}}^{L-1}\left(\sum_{n \forall\left(d_{k+l}^{b}=d_{k+l}^{n}\right)}^{e_{u}^{I}[r] \approx d^{u} \cdot R_{c c}(\Delta \tau) \cdot \operatorname{sinc}\left(\pi \Delta f T_{R}\right) \cdot} \mathrm{A}_{\mathrm{n}}^{2} \cos \left(2 \pi f_{n} \Delta \tau\right) \cos \left(\pi \Delta f 2(k+l) T_{R}+\Delta \theta\right)\right.
\end{gathered}
$$

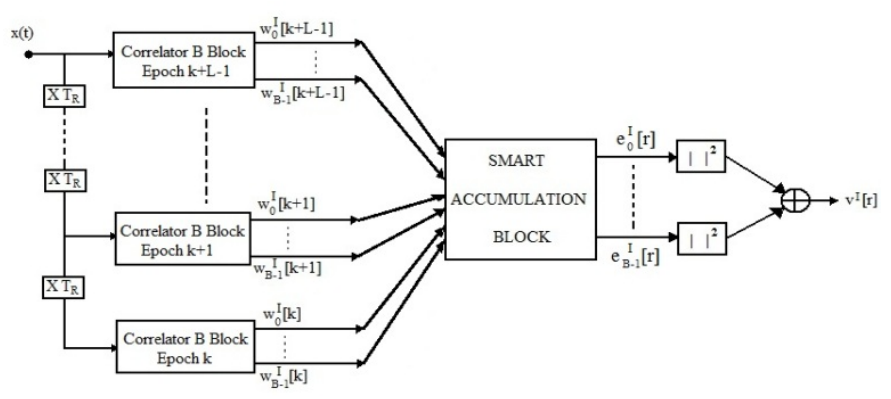

Figure 11: Correlator $\mathrm{C}$ block scheme

- X: Duration in PRN code periods of a transmitted symbol in a subcarrier ( $\mathrm{X}=1$ in Figure 3$)$.

- L: Number of accumulated correlator B block outputs. Maximum value of $\mathrm{L}$ is set by the group symbol length: $\mathrm{L}_{\max } \cdot \mathrm{X}=[\mathrm{t} 2, \mathrm{t} 1]$.

- $\mathrm{e}_{\mathrm{u}}[\mathrm{r}]$ : Coherent summation of the $\mathrm{w}_{\mathrm{b}}{ }^{\mathrm{I}}[\mathrm{k}+\mathrm{l}]$ terms carrying the data symbol $\mathrm{d}_{\mathrm{u}}$ at epochs $\mathrm{k}, \ldots, \mathrm{k}+\mathrm{L}-1$

- $\quad r$ : the epoch enclosing period $[\mathrm{k}, \mathrm{k}+\mathrm{L}-1]$

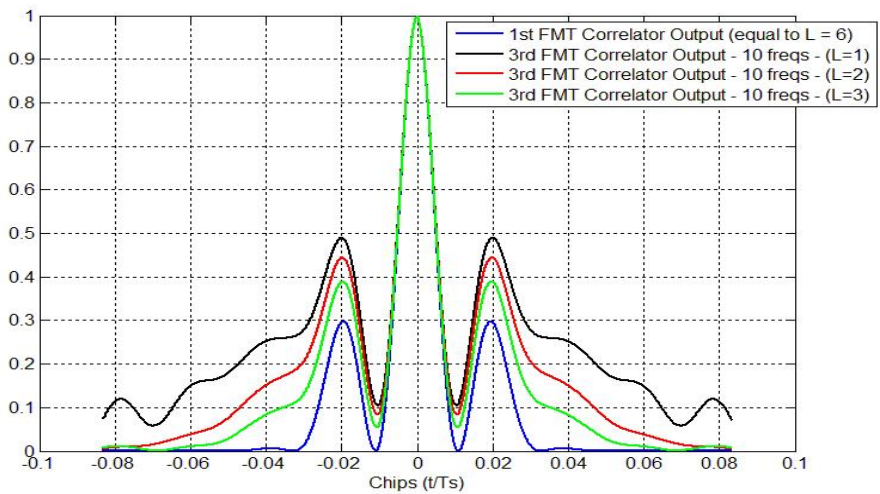

Figure 12: Normalized non-coherent correlator $\mathrm{C}$ output

TABLE II. NON-COHERENT OUTPUT MAIN LOBE TO SECONDARY LOBE RATIO FOR DIFFERENT SYMBOL GROUP CONFIGURATIONS OF THE CORRELATOR C BLOCK

\begin{tabular}{|c|c|}
\hline $\begin{array}{c}\text { Number of accumulated outputs } \\
\text { of the } \mathbf{2}^{\text {nd }} \text { FMT correlator }(\mathbf{L})\end{array}$ & $\begin{array}{c}\text { Ratio between the main and } \\
\text { secondary lobes (dB) }\end{array}$ \\
\hline 1 & 6.1981 \\
\hline 2 & 7.04 \\
\hline 3 & 8.1834 \\
\hline 6 & 10.5285 \\
\hline
\end{tabular}

Equation (28) highlights that term $\cos \left(\pi \Delta f 2(k+l) T_{R}+\Delta \theta\right)$ is a disturbing element since it distortions the original weight, $A_{n}$, associated to each subcarrier. Therefore, correlator $C$ can only be used either when $\Delta \mathrm{f}$ and $\Delta \theta$ are perfectly estimated $(\approx 0)$ or when term $\pi \Delta f 2 k T_{R}+\Delta \theta$ has almost the same value as $\pi \Delta f 2(k+L-1) T_{R}+\Delta \theta$. For now, we assume that one of the two assumptions is verified and later, in the acquisition and in the tracking sections, they will be discussed. Then, $\mathrm{e}_{\mathrm{u}}^{\mathrm{I}}[\mathrm{r}]$ is:

$$
\begin{gathered}
e_{u}^{I}[r] \approx d^{u} \cdot R_{c c}(\Delta \tau) \cdot \operatorname{sinc}\left(\pi \Delta f T_{R}\right) \cdot \cos \left(\pi \Delta f(k+L / 2) T_{R}+\Delta \theta\right) \\
\cdot \sum_{\substack{l=0 \\
b \forall\left(d^{u}=d_{k+1}^{b}\right)}}^{L-1}\left(\sum_{n \forall\left(d_{k+l}^{b}=d_{k+l}^{n}\right)} A_{\mathrm{n}}^{2} \cos \left(2 \pi f_{n} \Delta \tau\right)\right)
\end{gathered}
$$


And $\mathrm{e}^{\mathrm{Q}}{ }_{\mathrm{u}}[\mathrm{r}]$ has the same expression as $\mathrm{e}_{\mathrm{u}}^{\mathrm{I}}[\mathrm{r}]$ but with a sine instead of a cosine for the $\pi \Delta f(k+L / 2) T_{R}+\Delta \theta$ term.

Figure 12 shows the correlator $\mathrm{C}$ normalized non-coherent output: $\mathrm{v}^{\mathrm{I}}[\mathrm{k}]$ and $\mathrm{v}^{\mathrm{Q}}[\mathrm{k}]$ expressions addition when assuming perfect signal carrier frequency estimation $(\Delta \mathrm{f} \approx 0)$. Correlator $\mathrm{C}$ output depends on the value of $\mathrm{L}$ and on the value of $\mathrm{T}$. Therefore, Figure 12 shows different configurations of $\mathrm{L}$ for a fixed value of $\mathrm{T}=10$ subcarriers $(5$ positive subcarrier frequencies and 5 negative subcarriers frequencies in order to respect the subcarrier data symbol symmetry condition of the $0 \mathrm{~Hz}$ frequency axis). Each group of subcarriers is set to transport the same data symbol for only 1 PRN code period $(\mathrm{X}=1)$ in order to maximize the FMT signal frequency diversity. Therefore, $\mathrm{L}_{\max }$ is set to $\mathrm{M} / \mathrm{T}$ in order to allocate to any $3^{\text {rd }}$ data distribution data symbol the same energy as the energy of a $2^{\text {nd }}$ data distribution data symbol when $X=1$ as well. In this case, $\mathrm{L}=\{1,2,3,6\}$ in order to obtain a constant $\mathrm{T}$ value for any group of subcarriers carrying the same symbol at a given epoch $\mathrm{k}$. Note that $\mathrm{L}=1$ configuration correlator $\mathrm{C}$ output is equivalent to correlator $\mathrm{B}$ output and that $\mathrm{L}=6$ configuration correlator $\mathrm{C}$ output is equivalent to correlator $\mathrm{A}$ output. Numerical values used to represent Figure 12 are given in section II.C.

As was observed from Figure 10, Figure 12 shows that regardless of the $\mathrm{L}$ configuration, the correlation function main peak has always the same width. The ratio between the main lobe and the secondary lobe of different configurations is shown in TABLE II.

\section{E. FFT-based correlator}

The general receiver structure of a multi-carrier (MC) signal such as an OFDM signal or a FMT signal is based on FFT blocks [6]. Therefore, the question whether an FMT signal specially designed as a GNSS signal should use this same type of receiver structures is raised.

The main idea behind using an FFT block at reception is to apply a FFT transformation to the incoming signal and directly obtain in each component of the resulting FFT vector the symbol transmitted by one and only one subcarrier of the signal [6]. In other words, one component of the FFT vector represents the value transmitted by one individual subcarrier. This operation is called "channeling" and presents a reduction of the receiver's complexity: the receiver substitutes the signal multiplication by a given subcarrier plus the application of a low-pass filter intended to recover the single subcarrier value by the application of a FFT operation; where one such multiplication and filter are required for each individual signal subcarrier and only one FFT is necessary for all subcarriers.

An analysis of the number of operations necessary for a FFT-based type of correlator and the number of operations necessary for the correlators presented in this paper should determine if this complexity reduction for the type of FMT signal presented in this paper is achieved.

In order to correctly apply the channeling operation, the symbol value of each subcarrier must be constant during the
FFT application time. This fact implies that only one symbol of each subcarrier can be channelized at the same time. Therefore, a FFT-based correlator needs to channelize $\mathrm{G}$ times the FMT signal described in this paper (MC-DS-CDMA signal), where $G$ is the length of the spreading code, in order to recover the entire spreading code of each subcarrier: one chip of each subcarrier is recovered at each FFT application at a different position of the FFT output vector. Afterwards, each recovered PRN code has to be fed to a classical correlator (local replica, integration time). To sum up, a MC-DS-CDMA signal needs to apply $G$ times the FFT operation on the incoming baseband signal to obtain the entire spreading code, to individually multiply each subcarrier spreading code by its own local replica (M.G multiplications where $\mathrm{M}$ is the number of subcarriers) and to individually accumulate the multiplication result $(\mathrm{M} \cdot(\mathrm{G}-1)$ additions).

This operations list is compared with the correlators operations list proposed on this paper. The Proposed correlators only need to multiply the incoming total FMT baseband signal to each subcarrier local replica $(M \cdot G$ multiplications) and to individually accumulate subcarrier multiplication result (M.(G-1) additions). In fact, each correlator is also a low-pass filter which isolates the desired subcarrier whereas the local replica multiplication translates the subcarrier to the baseband frequencies. Moreover, the different circular shifts of each subcarrier also contribute to eliminate the undesired subcarriers at the correlator output.

Therefore, previous explanation shows that the proposed correlators are less complex than a FFT-based correlator since a FFT-based correlator executes G FFT additional operations. However, an advantage of using a FFT for channelization is the removal of the cross-correlation between different subcarriers PRN codes. But, given the small number of FMT signal subcarriers and the good cross-correlation properties of the PRN code sequences due to a large G (>1023 chips), we recommend to use the proposed correlator blocks without implementing FFT blocks. Additionally, the proposed correlator blocks in this paper are not so constrained by the sampling frequency which is a factor that could improve the complexity of a FFT-based correlator.

\section{ACQUISITION AND SYNCHRO-SYMBOL PROCESS}

In this section, the acquisition process, the synchro-symbol process as well as the particularities of each data distribution are commented. Moreover, a first preliminary acquisition performance comparison is made between the proposed data distributions and their correlators. Finally, the Doppler ambiguity phenomenon and its mitigation are described.

\section{A. Acquisition block and acqusition strategies}

The acquisition block of the FMT signal defined in this paper is the same as a typical acquisition block of a classical GNSS signal [7], where the only difference is the search range parameters and the correlator block which is replaced by one of the proposed FMT signal correlator blocks on this paper. The acquisition strategy proposed to acquire the FMT signal is the classical one and is well documented in the literature [7]. 


\section{B. Acquisition for the different data distributions}

In addition to the pilot component, the data component can also be used to acquire the incoming signal. In this case, where the receiver decides to use both components instead of only using the pilot, the receiver must proceed differently depending on the implemented data distribution. The main difference between the different data distributions on the acquisition process is whether the starting point of the transmitted symbol groups has to be known or not. Graphical examples of the coherent and non-coherent additions of the correlator outputs showing this difference are given.

$1^{\text {st }}$ and $2^{\text {nd }}$ data distributions do not need to know the group symbol starting point because setting the correlator A integration time to $1 \mathrm{PRN}$ code period $(\mathrm{X}=1)$ guarantees that the non-coherent correlator output presented in Figure 7 is always found. Figure 13 shows the correlator A coherent and non-coherent operations of the acquisition process.

$3^{\text {rd }}$ data distribution when using correlator $\mathrm{B}$ does not either need to know the starting point of the transmitted symbol groups if $\mathrm{X}=1$ and if the group symbol structure is symmetric in two senses: symmetric inside the transmitted group symbol from 1 PRN code to the next one, and symmetric outside the transmitted group symbol, from transmitted symbol group to transmitted symbol group. Figure 14 shows the correlator B coherent and non-coherent operations of the acquisition process.

Finally, $3^{\text {rd }}$ data distribution when using correlator $\mathrm{C}$ does need to know the starting point of the transmitted symbol groups since $\mathrm{X}$ is different from 1 by definition. Figure $15 \mathrm{a}$ and Figure $15 \mathrm{~b}$ show the correlator $\mathrm{C}$ coherent and noncoherent operations of the acquisition process and why the symbol group starting point is required: if the starting point is not known, we cannot guarantee that the smart accumulation box coherently adds in time the same transmitted data symbols. Therefore, since in the acquisition process the starting point is not known, the chip bin search is L times the chip bin search of correlator A and B acquisition processes.

One important constraint about correlator $\mathrm{C}$ acquisition process is the necessity of having a constant term $\cos \left(\pi \Delta f 2(k+l) T_{R}+\Delta \theta\right)$ for $l \varepsilon[0, L-1]$. If this constraint is not respected, the different added subcarrier frequencies by the accumulation smart block in different instants of time $(l)$ have different weights (see equation (28)) and thus Figure 12 original correlation functions are not recovered. In fact, this constraint imposes the frequency bin width $(\Delta \mathrm{f})$ since term $\pi \Delta f 2(k+l) T_{R}$ can be interpreted as the evolution in time of the signal carrier phase estimation error whereas term $\Delta \theta$ represents the initial signal carrier phase estimation error which is constant for the period $\left[0,(L-1) T_{R}\right]$.

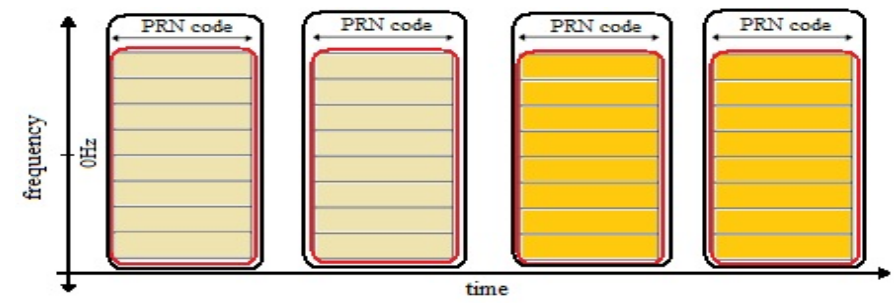

Figure 13: Coherent and non-coherent operations of the acquisition process of the $1^{\text {st }}$ and $2^{\text {nd }}$ data distributions

- Red squares: Coherent correlation area inside a noncoherent area.

- Black squares: Non-coherent correlation area

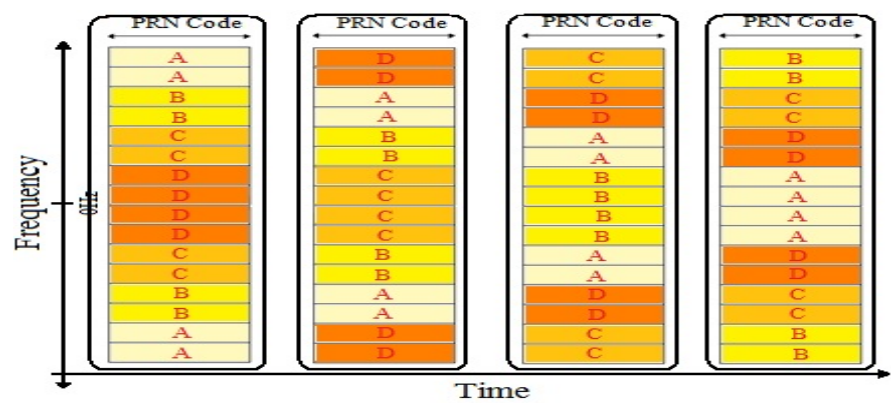

Figure 14: Coherent and non-coherent operations of the acquisition process of the $3^{\text {rd }}$ data distributions for the $1^{\text {st }}$ option correlator

- Same red letters: Coherent correlation area inside a non-coherent area.

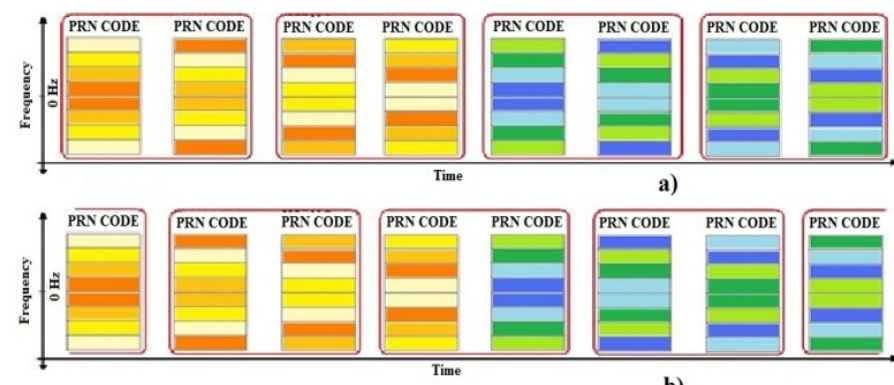

Figure 15: Coherent and non-coherent operations of the acquisition process of the $3^{\text {rd }}$ data distributions for the $2^{\text {nd }}$ option correlator

In order to analyze this constraint, we have to set the maximum difference of amplitude between subcarriers received at $\mathrm{l}=0$ and subcarriers received at $\mathrm{l}=\mathrm{L}-1$. Two values have been chosen in this paper, 0.8 and 0.9 , which means that the worst attenuation suffered by a subcarrier or group of subcarriers with respect to a subcarrier or group of subcarriers suffering the lowest attenuation is $0.8 / 0.9$. The worst situation is found when $2 \pi \Delta f 2 k T_{R}+\Delta \theta=2 \pi n$ with $n \in \mathrm{Z}$. Setting $\cos \left(\pi \Delta f 2(L-1) T_{R}\right)=0.8 / 0.9$ provides the value of $\Delta \mathrm{f}$ :

- $\cos \left(\pi \Delta f 2(L-1) T_{R}\right)=0.8 \rightarrow \Delta f=1 /\left[5(L-1) \cdot 2 T_{R}\right]$

- $\cos \left(\pi \Delta f 2(L-1) T_{R}\right)=0.9 \rightarrow \Delta f=1 /\left[7(L-1) \cdot 2 T_{R}\right]$

Finally, this value is compared to the $\Delta \mathrm{f}$ value required for correlators $\mathrm{A}$ or $\mathrm{B}, \Delta f=1 /\left(2 T_{R}\right)$. Therefore, correlator $\mathrm{C}$ 
requires about $5(\mathrm{~L}-1)$ or $7(\mathrm{~L}-1)$ times more frequency bins than correlators A or B.

To sum up, correlator $\mathrm{C}$ requires about $5(\mathrm{~L}-1) \mathrm{M}$ or $7(\mathrm{~L}-$ 1)M times the total number of bins of correlators $\mathrm{A}$ or $\mathrm{B}$.

\section{Acquisition preliminary performances}

Two types of figures are shown in order to preliminary compare the acquisition performances of the different correlator blocks. Figure 16 shows the output of each proposed correlator block in the presence of AWG noise as a function of the signal code delay estimation error. $3^{\text {rd }}$ data distribution configurations (L, T) are the same as the ones presented for correlator $\mathrm{C}$ in Figure 12. Non-coherent integrations time is set to $24 \mathrm{PRN}$ code periods.

Figure 16 shows that the main lobe is more easily identified for correlator $\mathrm{A}$ than for correlator $\mathrm{B}$ and $\mathrm{C}$, and thus, correlator A has the best acquisition performance. This difference of acquisition performance depends on the quadratic noise term: for low $\mathrm{C} / \mathrm{N}_{0}$ this difference increases and for high $\mathrm{C} / \mathrm{N}_{0}$ this difference decreases.

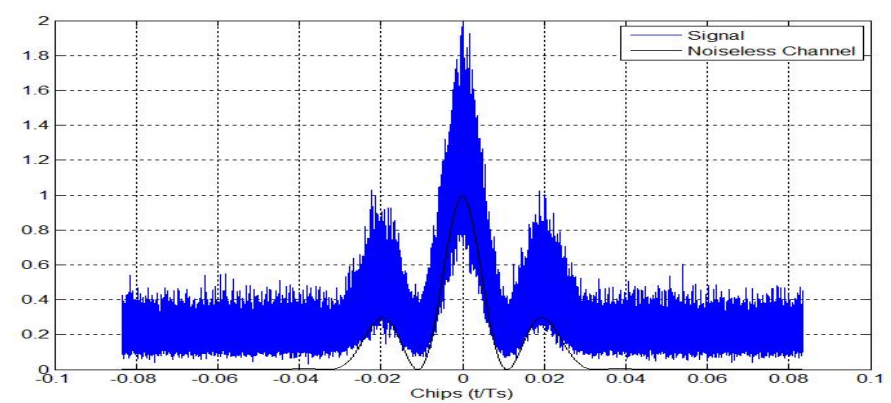

a)

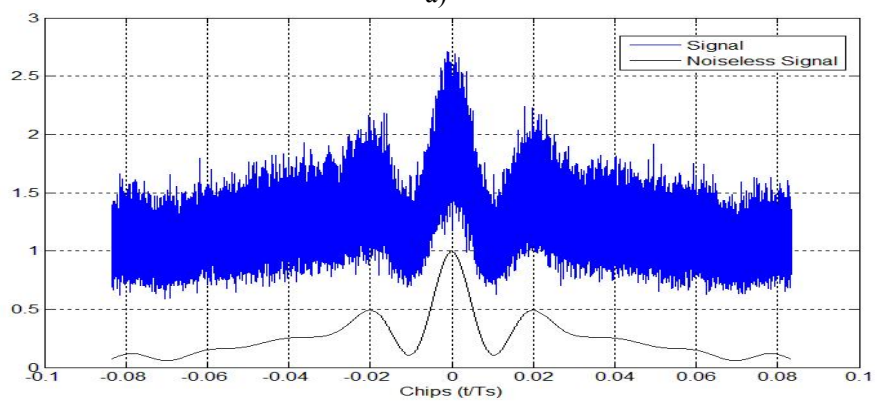

b)

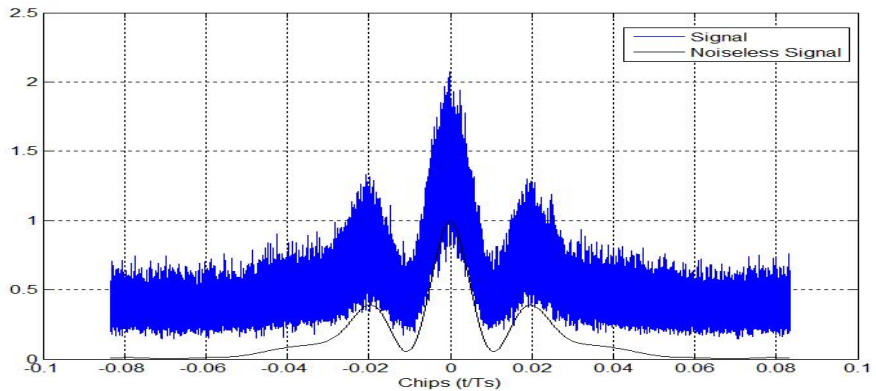

c)

Figure 16: Normalized correlator ouput vs signal code delay estimation error. $\mathrm{C} / \mathrm{N}_{0}=15 \mathrm{~dB}-\mathrm{Hz}$ and non_coh $=24 \mathrm{PRN}$. a)

Correlator A $(\operatorname{coh}=1 P R N)$. b) Correlator $B(L=1, \operatorname{coh}=1 P R N)$. c) Correlator $C(L=3, \operatorname{coh}=3 P R N)$.
Figure 17 shows the correlators output average power of their main lobe with respect to their average power of their secondary lobe. The difference between the powers gives an indication of the probability of causing a false alarm event where the largest difference implies a lower false alarm probability.

Figure 17 shows that the largest difference of power is found for correlator $\mathrm{A}$ and the worst for correaltor $\mathrm{B}$. Moreover, it has been verified through other simulations that this difference between the average power of the main lobe and the average power of the secondary lobe also increases further for correlator $A$ than for correlator $B$ when the $C / \mathrm{N}_{0}$ increases. Therefore, Figure 17 also shows that correlator A provides a better acquisition performance than correlator $\mathrm{B}$ since the probability of having a false alarm is lower for correlator A.

To sum up, correlator A presents the best acquisition performance with the smallest space search (number of bins) whereas correlators $\mathrm{B}$ and $\mathrm{C}$ present worse acquisition performance and a larger space search for correlator C.

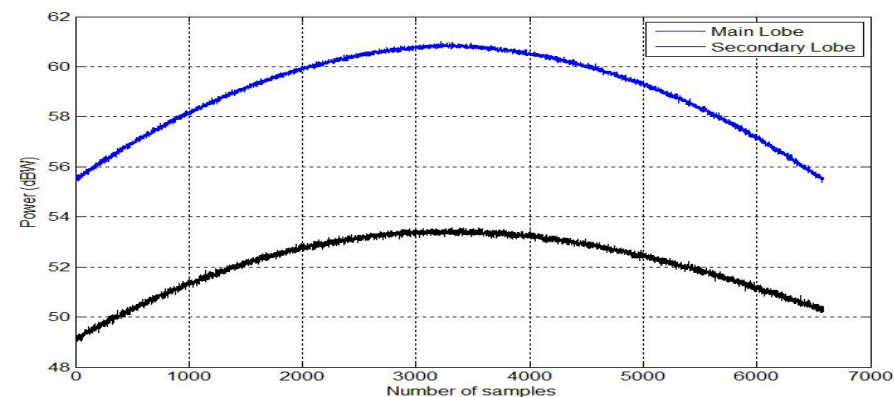

a)

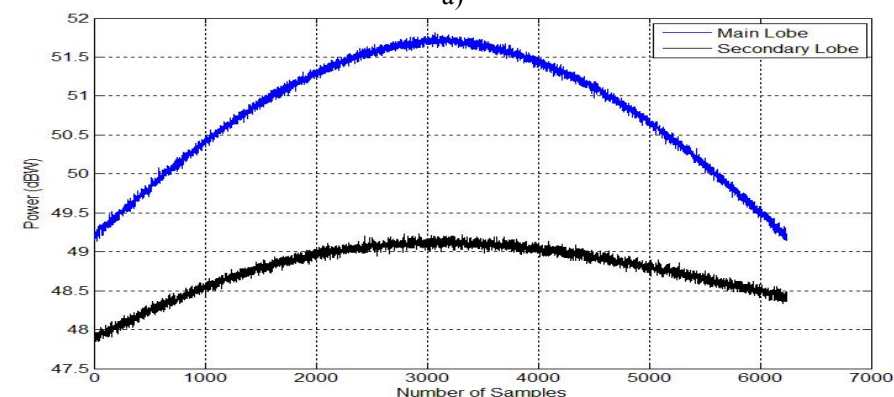

b)

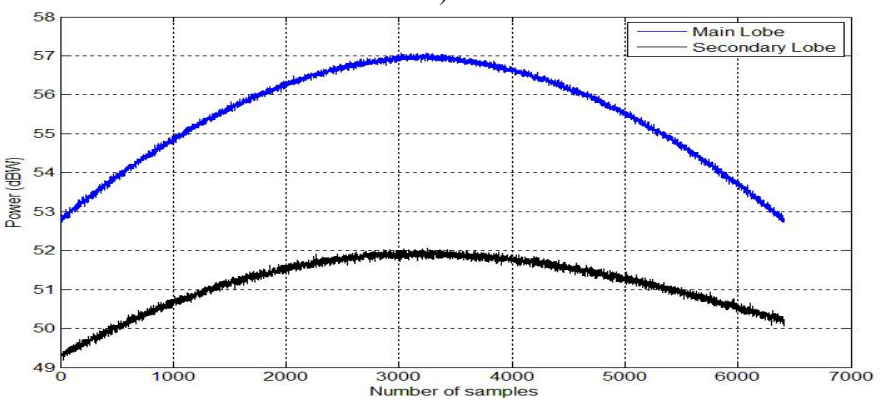

c)

Figure 17: Correlator output main lobe and secondary lobe average power. $\mathrm{C} / \mathrm{N}_{0}=15 \mathrm{~dB}-\mathrm{Hz}$ and non $\operatorname{coh}=24 \mathrm{PRN}$. a) Correlator A $(\operatorname{coh}=1 \mathrm{PRN})$. b) Correaltor $\mathrm{B}^{-}(\mathrm{L}=1, \operatorname{coh}=1 \mathrm{PRN})$. c) Correlator $\mathrm{C}(\mathrm{L}=3, \operatorname{coh}=3 \mathrm{PRN})$. 
Besides, correlator $\mathrm{B}$ acquisition performance (or $3^{\text {rd }}$ data distribution) depends on the number of adjacent subcarriers transporting the same symbol, $\mathrm{T}$, where this performance increases along with $\mathrm{T}$ (correlator $\mathrm{B}$ becomes more similar to correlator A). Additionally, since correlator $\mathrm{C}$ presents better acquisition performance than correlator B (reaching the same acquisition performance as correlator A if the FMT signal is well designed and $\mathrm{L}=\mathrm{L}_{\max }$ ), for the $3^{\text {rd }}$ data distribution on low $\mathrm{C} / \mathrm{N}_{0}$ environments an alternative acquisition strategy can be proposed: a first rough acquisition with correlator $\mathrm{B}$ and second more precise one with correlator $\mathrm{C}$.

Finally, remember that the acquisition process could only be applied on the pilot component and thus the data distribution does not play any role on the acquisition process.

\section{Synchro-symbol process}

The $2^{\text {nd }}$ data distribution does not need to execute the synchro-symbol process if the FMT signal is smartly designed: one data symbol exactly spans 1 PRN code of the data component as well as 1 PRN code of the pilot component. In this case, the acquisition process already provides the symbol synchronization.

In the case of the $3^{\text {rd }}$ data distribution, the receiver must find the group symbol starting point in order to obtain the data symbol synchronization. The synchro-symbol process could simply consist in applying the coherent and non-coherent additions of Figure 15 and in choosing the output with the largest value as the group symbol starting point. Additionally, the pilot component could implement an additional code spanning the group symbol length in order to help with the symbol synchronization process.

\section{E. Doppler ambiguity}

The Doppler ambiguity phenomenon refers to the situation where the subcarrier $f_{n}$ of the received signal locks with the subcarrier $f_{m}$ of the local replica due to a shift of the received signal central frequency by the Doppler effect (motion of receiver and/or the emitter). Besides, this lock can also be propagated to the remaining received subcarriers which have a frequency match with the remaining local generated subcarriers (ex: $f_{n+1}$ of the received signal with the $f_{m+1}$ of the local replica, etc...). Figure 18 shows a graphical example.

The false lock between a local replica subcarrier and a received signal subcarrier only occurs when the two subcarriers implement the same PRN code. More importantly, the catastrophic propagation only occurs when all the subcarriers share the same PRN code. Therefore, one possible solution to mitigate this effect consists in transmitting one different PRN code for each subcarrier. However, the total number of required PRN codes rapidly becomes too large when taking into account the different components of a signal (at least one pilot and one data component) and the number of satellites of the constellation.

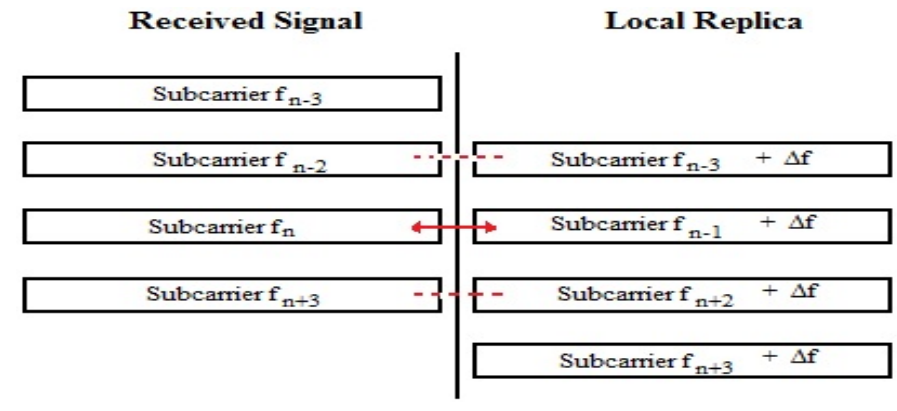

Figure 18: Doppler Ambiguity effect

- $\Delta \mathrm{f}$ : Frequency Doppler shift on the central frequency of the FMT signal

- $\mathrm{f}_{\mathrm{n}}=\mathrm{f}_{\mathrm{n}-1}+\Delta \mathrm{f}$

- Red arrow: False lock between a subcarrier of the local replica and of the received signal.

- Red dot-line: Possible propagation locks between local replica subcarriers and received subcarriers.

The solution proposed in this paper consists in associating one PRN code to all the subcarriers of one satellite component (as it is classically done) but associating different circularly shifted versions of the PRN code to each subcarrier. This association is made applying a non-uniform mapping, $F(n)$, which is discussed in more detail in section V.F but the main idea is presented next: the code delay required to lock subcarriers $n\left(f_{n}\right)$ and $m\left(f_{m}+\Delta f\right)$ is different from the code delay required to lock subcarriers $n+1\left(f_{n+1}\right)$ and $m+1\left(f_{m+1}+\right.$ $\Delta \mathrm{f}$ ) with $n, m \in \mathrm{Z}$. Therefore, although this non-uniform mapping does not prevent the initial false lock between a received subcarrier and a local replica subcarrier, it avoids the propagation of the lock to other subcarriers, meaning that the Doppler ambiguity effect is vastly mitigated.

A mathematical explanation of this mitigation effect is presented next where $\mathrm{x}_{\mathrm{k}}(\mathrm{t})$ is the equivalent baseband received signal at epoch $\mathrm{k}, \mathrm{r}_{\mathrm{k}}^{\mathrm{l}}(\mathrm{t})$ is the equivalent baseband generated local replica at epoch $\mathrm{k}$ and $\mathrm{A}_{\mathrm{n}}$ is assumed to be 1:

$$
\begin{gathered}
x_{k}(t)=\sum_{n \in D(M)} c_{F(n)}(t) \cdot e^{j 2 \pi\left(f_{n}+\Delta f\right) t} \\
r_{k}(t)=\sum_{n \in D(M)} c_{F(n)}\left(t-\tau_{0}\right) \cdot e^{-j 2 \pi f_{n}\left(t-\tau_{0}\right)}
\end{gathered}
$$

The relationships of frequency between the subcarriers and the Doppler frequency are:

$$
f_{n}=f_{n-1}+\Delta f_{D O P} \quad n \in D(M)
$$

Using correlator A without loss of generality:

$$
\begin{gathered}
v^{I}[k]=\int_{-T_{R} / 2}^{T_{R} / 2} x_{k}(t) \cdot r_{k}(t) d t \\
v^{I}[k]= \\
\int_{-T_{R} / 2}^{T_{R} / 2} \sum_{n \in D(M)} \sum_{u \in D(M)} c_{F(n)}(t) c_{F(u)}\left(t+\tau_{0}\right) \cdot e^{j 2 \pi\left(f_{n}+\Delta f\right) t} \cdot e^{-j 2 \pi f_{u}\left(t+\tau_{0}\right)} d t
\end{gathered}
$$


Pass-band terms are removed by the integration. Therefore, from equation (32), the correlator output is approximated as:

$$
v^{I}[k]=\sum_{(n, n-1) \epsilon D(M)} R_{F(n) F(n-1)}\left(\tau_{0}\right) \cdot e^{j 2 \pi f_{n} \tau_{0}}
$$

Equation (35) shows that the only remaining terms are the crossed correlated subcarriers as was expected. However, due to the imposed non-uniform mapping, $F(n)$, all this terms are null except for one because all the correlations functions are zero except for the one that fulfills the following equality:

$$
F(n)-F(n-1)=\tau_{0}
$$

Therefore, $\mathrm{v}^{\mathrm{I}}[\mathrm{k}]$ is equal to:

$$
v^{I}[k]=R_{F(n) F(n-1)}\left(\tau_{0}\right) \cdot e^{j 2 \pi f_{n} \tau_{0}}
$$

Equation (37) shows that the FMT Doppler ambiguity effect is greatly mitigated and thus that the acquisition false lock probability due to the FMT Doppler ambiguity effect is very low.

\section{F. PRN code non-uniform mapping construction}

The characteristics allowing the correct generation of the non-uniform mapping, $F(n)$, between circular shifts of the same PRN code and subcarriers are presented next.

First, for obvious reasons, F(n) must be injective: it never maps distinct elements of its domain to the same element of its codomain (all the circular shifts are unique):

$$
F\left(n_{1}\right) \neq F\left(n_{2}\right) \quad\left(n_{1}, n_{2}\right) \in D(M) \mid n_{1} \neq n_{2}
$$

Second, the difference between any pair of circular shifts, $\mathrm{F}\left(\mathrm{n}_{2}\right), \mathrm{F}\left(\mathrm{n}_{1}\right)$, must be different from any other pair of circular shifts, $F\left(n_{4}\right), F\left(n_{3}\right)$. Therefore, in the case where the subcarriers carrying circular shifts $\mathrm{F}\left(\mathrm{n}_{2}\right)$ and $\mathrm{F}\left(\mathrm{n}_{1}\right)$ are falsely locked due to the Doppler effect (match in frequency and code delay equal to $\left.\mathrm{F}\left(\mathrm{n}_{2}\right)-\mathrm{F}\left(\mathrm{n}_{1}\right)\right)$, even if subcarriers $\mathrm{n}_{4}\left(\mathrm{~F}\left(\mathrm{n}_{4}\right)\right)$ and $\mathrm{n}_{3}\left(\mathrm{~F}\left(\mathrm{n}_{3}\right)\right)$ are matched in frequency, their required code delay $\left(\mathrm{F}\left(\mathrm{n}_{4}\right)-\mathrm{F}\left(\mathrm{n}_{3}\right)\right)$ will be different.

$$
\begin{gathered}
\bmod \left(F\left(n_{1}\right)-F\left(n_{2}\right), G\right) \neq \bmod \left(F\left(n_{1}\right)-F\left(n_{3}\right), G\right) \\
\left(n_{1}, n_{2}, n_{3}\right) \in D(M) \mid n_{1} \neq n_{2} \neq n_{3} \\
\bmod \left(F\left(n_{1}\right)-F\left(n_{2}\right), G\right) \neq \bmod \left(F\left(n_{3}\right)-F\left(n_{4}\right), G\right) \\
\left(n_{1}, n_{2}, n_{3}, n_{4}\right) \in D(M) \mid n_{1} \neq n_{2} \neq n_{3} \neq n_{4}
\end{gathered}
$$

However, this condition is quite restrictive and cannot be accomplished if the number of PRN code chips $(\mathrm{G})$ is small and the number of subcarriers is high (M). Therefore, we can relax this condition without introducing any degradation in the solution by imposing equations (39) and (40) only when absolute frequency difference between $f_{n 2}$ and $f_{n 1}$ is the same as the absolute frequency difference between $f_{n 4}$ and $f_{n 3}$. If this difference is not the same, when subcarrier $f_{n 2}$ is falsely locked with subcarrier $f_{n 1}$, subcarriers $f_{n 4}$ and $f_{n 3}$ cannot lock because they do not match in frequency:

$$
\begin{gathered}
\bmod \left(F\left(n_{1}\right)-F\left(n_{2}\right), G\right) \neq \bmod \left(F\left(n_{3}\right)-F\left(n_{4}\right), G\right) \\
\text { and }\left|f_{n 2}-f_{n 1}\right|=\left|f_{n 4}-f_{n 3}\right| \\
\left(n_{1}, n_{2}, n_{3}, n_{4}\right) \in D(M) \mid n_{1} \neq n_{2} \neq n_{3} \neq n_{4}
\end{gathered}
$$

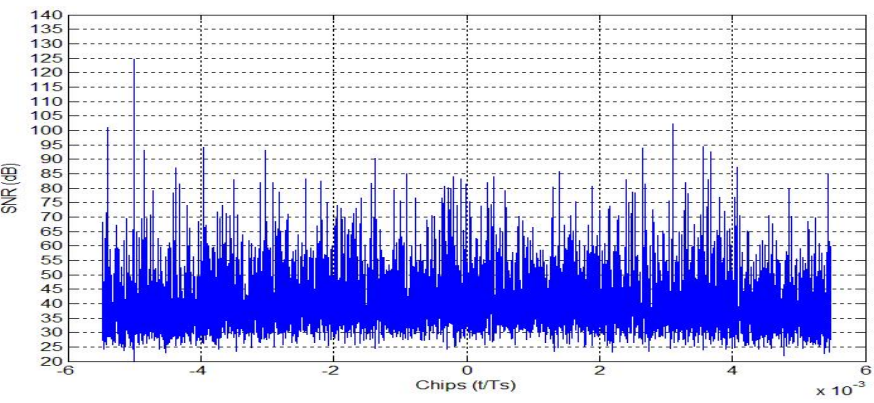

a)

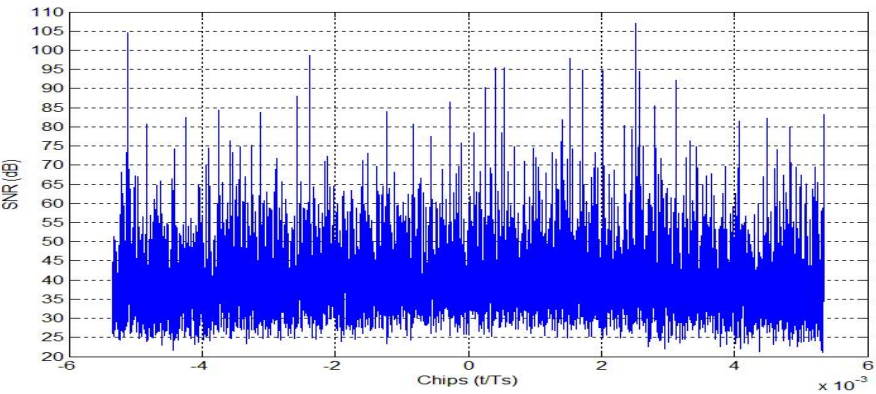

b)

Figure 19: Correlators output main lobe $S N R . C / N_{0}=15 \mathrm{~dB}-\mathrm{Hz}$, coherent integration time $=3 P R N$ and non-coherent integration additions = 1. a) Correlator A. b) Correlator C

\section{TRACKING PROCESS}

The tracking of a FMT signal is made using the classical DLL, FLL and PLL structures with the desired discriminators [8]. The differences with respect to a BPSK/BOC modulation are the correlator block, which must be chosen among the 3 proposed ones, and the typical parameters values such as the DLL correlator spacing and coherent integration time.

In the tracking process, as opposite to the acquisition process, the symbol group starting point is always known since the syncro-symbol process is already achieved. This means that correlator $\mathrm{C}$ can be used without constrains and, since correlator $\mathrm{C}$ provides exactly the same non-coherent output as correlator $\mathrm{A}$, it can be concluded that the $1^{\text {st }}, 2^{\text {nd }}$ and $3^{\text {rd }}$ data distributions provide the same tracking performance. The only difference is found on the coherent integration time: whereas correlator $\mathrm{C}$ coherent integration time has to be a multiple of the group symbol length, correlator A coherent integration time has to be a multiple of the data symbol length.

However, a tracking performance preliminary analysis for the different correlators when using a shorter integration time than the group symbol length is presented. Figure 19 shows the SNR at the correlator output main lobe in presence of AWG noise: correlator $\mathrm{A}$ with a coherent integration time of 3 PRN code periods, and correlator $\mathrm{C}$ with an equivalent coherent integration time equal to 3 PRN code periods (real coherent integration time of 1 PRN code period with $\mathrm{L}=3$ ).

Figure 19 shows that the SNR at the correlator output main lobe is higher for correlator A than for correlator C. In fact, this SNR difference grows along the $\mathrm{C} / \mathrm{N}_{0}$ decrease and the decrease of the total number of added coherent integrations (L). The largest difference is found for $\mathrm{L}=1$ (correlator $\mathrm{B}$ ). 


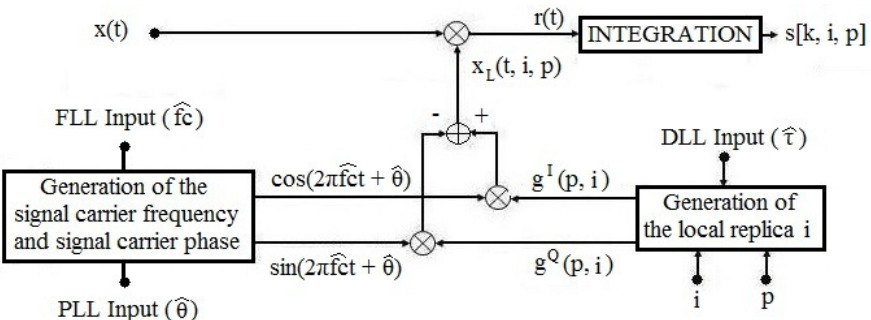

Figure 20: Partial correlator block general scheme of the $p^{\text {th }}$ part of the $i^{\text {th }}$ data symbol of the $k^{\text {th }}$ symbol group

- $\quad \mathrm{k}$ : indicates transmitted symbol group

- $\mathrm{i}$ : indicates data symbol inside the $\mathrm{k}^{\text {th }}$ transmitted symbol group $i \quad[0, I-1]$

- I: Number of data symbols inside a group symbol

- $\mathrm{p}$ : indicates part of the $\mathrm{i}^{\text {th }}$ data symbol inside the $\mathrm{k}^{\text {th }}$ transmitted group symbol, $p \quad[0, P-1]$

- P: Number of parts of the data symbol transmitted in different subcarriers

- $\mathrm{T}_{\mathrm{X}}$ : Duration of a data symbol part transmitted on a subcarrier $\left(T_{D} / T_{X}=P \mid P \in \mathrm{Z}\right)$

- $T_{D}$ : Data symbol duration (multiple of $T_{R}$ )

- $\mathrm{p}_{\mathrm{T}}(\mathrm{t})$ : Rectangular pulse of duration $\mathrm{T}$

- $\mathrm{A}_{(\mathrm{i}, \mathrm{p})}$ : Amplitude of the subcarrier transmitting the $\mathrm{p}^{\text {th }}$ part of the $\mathrm{i}^{\text {th }}$ data symbol with $A_{i, p}=A_{\bmod (i+p, M)}$

- $\mathrm{f}_{(\mathrm{i}, \mathrm{p})}$ : Frequency of the subcarrier transmitting the $\mathrm{p}^{\text {th }}$ part of the $\mathrm{i}^{\text {th }}$ data symbol with $f_{i, p}=f_{\bmod (i+p, M)}$

- $\mathrm{x}^{\mathrm{L}}(\mathrm{t}, \mathrm{i}, \mathrm{p})$ : generated local replica for the $\mathrm{p}^{\text {th }}$ part of the $\mathrm{i}^{\text {th }}$ data symbol inside the $\mathrm{k}^{\text {th }}$ group symbol

\section{DEMODULATION PROCESS}

In this section, the demodulator blocks of the $2^{\text {nd }}$ to $4^{\text {th }}$ data distributions are presented.

\section{A. Demodulator block of the $2^{\text {nd }}$ data distribution}

The $2^{\text {nd }}$ data distribution demodulator block has exactly the same structure as the coherent in-phase correlator A block (see Figure 6). Therefore, the estimated data symbol at epoch $\mathrm{k}$ is given by expression $\mathrm{v}^{\mathrm{I}}[\mathrm{k}]$ (see equation (21)).

\section{B. Demodulator block of the $3^{\text {rd }}$ data distribution}

The $3^{\text {rd }}$ data distribution demodulator block has the same structure as the in-phase corraltor $\mathrm{C}$ block with $\mathrm{L}=\mathrm{L}_{\max }$ except for the smart addition block which is removed. In this case, since more than one symbol is broadcasted at the same time, at epoch $r$ the estimated data symbols are given by expressions $\mathrm{e}_{\mathrm{u}}^{\mathrm{I}}[\mathrm{r}]$ (see equation (29)).

This demodulator block should provide better demodulation performance than the $2^{\text {nd }}$ data distribution demodulator block since the $3^{\text {rd }}$ data distribution has a larger time diversity.

\section{Demodulator block of the $4^{\text {th }}$ data distribution}

The $4^{\text {th }}$ data distribution demodulator block and the justification of why $T_{x}$ must be a multiple of the PRN code period are presented next. The $4^{\text {th }}$ data distribution demodulator block is made of I single data symbol demodulator blocks, one for each parallel transmitted symbol. Each single data symbol demodulator is responsible of demodulating only one data symbol from the transmitted symbol group and is made of $\mathrm{P}$ partial correlator blocks, one for each subcarrier carrying the data symbol, which are coherently added to accumulate the total data symbol energy.

Figure 20 shows the structure of the partial correlator applied to the subcarrier transporting the $\mathrm{p}^{\text {th }}$ data part of the $i^{\text {th }}$ data symbol of the $\mathrm{k}^{\text {th }}$ parallel group of transmitted symbols. The mathematical expression of the partial in-phase and quadrature-phase local replicas are:

$$
\begin{aligned}
& g^{I}(p, i)=A_{(i, p)} \cdot p_{T x}\left(t-p T_{x}\right) \cdot c_{F(i)}(t-\hat{\tau}) \cdot \cos \left[2 \pi f_{(i, p)}(t-\hat{\tau})\right] \\
& g^{I}(p, i)=A_{(i, p)} \cdot p_{T x}\left(t-p T_{x}\right) \cdot c_{F(i)}(t-\hat{\tau}) \cdot \sin \left[2 \pi f_{(i, p)}(t-\hat{\tau})\right]
\end{aligned}
$$

General correlator output mathematical model, s[k,i,p] is:

$$
s[k, i, p]=\int_{(k-1) T_{D}+p T_{X}}^{(k-1) T_{D}+(p+1) T_{X}} x(t) \cdot x_{L}(t, i, p) \cdot d t
$$

Assuming a perfect signal carrier phase and signal carrier frequency estimation ( $\Delta \theta \approx 0$ and $\Delta f \approx 0$ ), equation (44) can be approximated for $\mathrm{p}_{0}$ part and $\mathrm{i}_{0}$ data symbol as:

$$
\begin{gathered}
s\left[k, i_{0}, p_{0}\right] \approx d_{i 0}^{k} \cdot \frac{\left(A_{i 0, p 0}\right)^{2}}{2} \cdot R_{F(i 0), p 0}(\Delta \tau) \cdot \cos \left(2 \pi f_{i 0, p 0} \Delta \tau\right) \\
R_{F(i 0), p 0}(\Delta \tau)=\int_{-T_{X} / 2}^{T_{X} / 2} c_{F(i 0), p 0}(t) c_{F(i 0), p 0}(t-\hat{\tau}) d t \\
c_{F(i 0), p}(t)=\sum_{m=p \cdot N_{T x}}^{(p+1) \cdot N_{T x}-1} c_{\bmod (m+F(i 0), M)} \cdot p_{T c}\left(t-m \cdot T_{c}\right)
\end{gathered}
$$

- $\mathrm{T}_{\mathrm{c}}$ : Chip time

- $\mathrm{N}_{\mathrm{Tx}}$ : Number of chips of the PRN code which are inside a symbol part length $\mathrm{T}_{\mathrm{X}}$

Note that depending on $\mathrm{T}_{\mathrm{X}}$ the values of $\mathrm{R}_{\mathrm{F}(\mathrm{i} 0), \mathrm{p} 0}(\Delta \tau)$ varies. If $\mathrm{T}_{\mathrm{x}}$ is a multiple of the PRN code length, $\mathrm{R}_{\mathrm{F}(\mathrm{i}), \mathrm{p} 0}(\Delta \tau)$ is the autocorrelation function of the PRN code. If $T_{x}$ is not a multiple of the PRN code length, $\mathrm{R}_{\mathrm{F}(\mathrm{n} 0), \mathrm{p} 0}(\Delta \tau)$ is not the autocorrelation function of the PRN code.

Figure 21 shows the structure of the demodulator of the $i^{\text {th }}$ data symbol of the $\mathrm{k}^{\text {th }}$ parallel group of transmitted symbols.

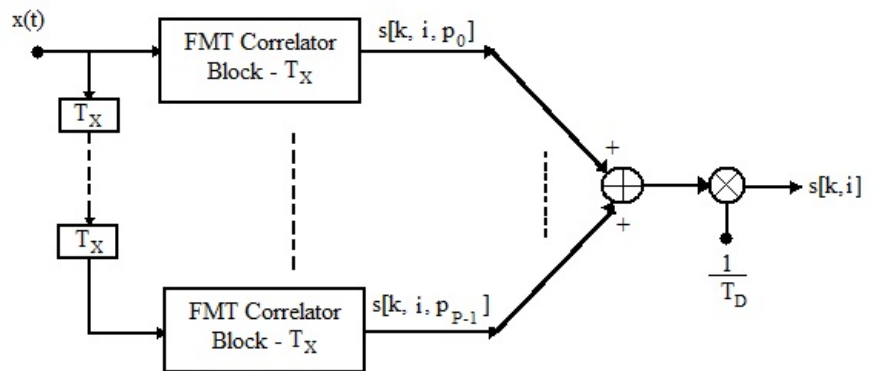

Figure 21: Single data symbol demodulator block scheme of the $i^{\text {th }}$ data symbol 
Assuming a good estimation of the signal code delay $(\Delta \tau \approx 0)$ and assuming for simplifications purposes $\left(A_{i 0, p 0}=1\right)$, $\mathrm{s}\left[\mathrm{k}, \mathrm{i}_{0}\right]$ can be expressed as:

$$
s\left[k, i_{0}\right] \approx \frac{d_{i 0}^{k}}{2 T_{D}} \cdot \sum_{p=0}^{P-1} R_{F(i 0), p}(\Delta \tau)
$$

However, expression (48) is incomplete since it does not take into account the other visible satellites of the constellation which are normally omitted when $T_{x}$ is a multiple of the PRN code period, but this is not the case here. Therefore, taking into account their influence, expression (48) becomes:

$$
\begin{aligned}
& s\left[k, i_{0}\right] \approx \frac{d_{i 0}^{k}}{2 T_{D}} \cdot \sum_{p=0}^{P-1} R_{F(i 0), p}(\Delta \tau)+ \\
&+\frac{d_{i 0}^{s, k}}{2 T_{D}} \cdot f\left(\Delta \theta_{s}, \Delta f_{s}\right) \sum_{s=0}^{S-1}\left(\sum_{p=0}^{P-1} R_{c F(i 0) c s F(i 0), p}(\Delta \tau) \cdot \cos \left(2 \pi f_{i 0, p} \Delta \tau_{s}\right)\right) \\
& R_{C F(n 0) c S F(i 0)}\left(\Delta \tau_{s}\right)=\int_{-T_{X} / 2}^{T_{X} / 2} c^{S}{ }_{F(i 0), p 0}(t) c_{F(i 0), p 0}\left(t-\Delta \tau_{s}\right) d t
\end{aligned}
$$

- $\quad \mathrm{S}$ : Number of visible satellites

- $\mathrm{d}^{\mathrm{s}, \mathrm{k}}{ }_{\mathrm{i} 0}$ : Value of the $\mathrm{i}_{0}$ data symbol of the $\mathrm{k}^{\text {th }}$ group symbol of the $s^{\text {th }}$ satellite

- $\Delta \mathrm{f}_{\mathrm{s}}$ : Frequency difference between the signal carrier frequency estimation of the desired satellite and the signal carrier frequency of the $\mathrm{s}^{\text {th }}$ satellite.

- $\Delta \theta_{\mathrm{s}}$ : Phase difference between the signal carrier phase estimation of the desired satellite and the signal carrier phase of the $\mathrm{s}^{\text {th }}$ satellite.

- $\Delta \tau_{\mathrm{s}}$ : Code dealy difference between the signal code delay estimation of the desired satellite and the signal code delay of the $s^{\text {th }}$ satellite.

- $\mathrm{f}\left(\Delta \mathrm{f}_{\mathrm{s}}, \Delta \theta_{\mathrm{s}}\right)$ : Partial contribution to the demodulator block output of the $\mathrm{s}^{\text {th }}$ satellite. $\Delta \mathrm{f}_{\mathrm{s}}$ and $\Delta \theta_{\mathrm{s}}$ are assumed to no vary for the different $p$ values.

$R_{c F(i 0) \operatorname{cs} F(i 0), p}\left(\Delta \tau_{s}\right)$ represents the partial correlation function between the $\mathrm{p}^{\text {th }}$ part PRN code of the $\mathrm{s}^{\text {th }}$ satellite received signal and the $p^{\text {th }}$ part PRN code of the generated local replica. This partial correlation is not 0 and the addition of all the partial correlations generates the complete correlation function between the two PRN codes, $R_{c F(i 0) \operatorname{csF}(i 0)}\left(\Delta \tau_{s}\right)$. Therefore, since the PRN codes are orthogonal by design, the complete correlation value is about 0 . However, in expression (50) each partial correlation is weighted by the cosine of the error between the estimated signal carrier frequency and the $\mathrm{s}^{\text {th }}$ satellite carrier frequency. Therefore, in order to remove the influence of the visible satellites, $T_{x}$ must be a multiple of $T_{R}$ so that the partial correlations become complete correlations. In this case, expression (50) is equal to:

$$
s\left[k, i_{0}\right] \approx \frac{d_{i 0}^{k}}{2}
$$

\section{SUMMARY}

A summary of the different data distributions and of the correlator blocks performances is provided in this section.

\begin{tabular}{|c|c|c|c|}
\multicolumn{2}{c}{ TABLE III. $\quad$ FMT DATA DISTRIBUTIONS SUMMARY } \\
\begin{tabular}{|c|c|c|c|}
\hline $\begin{array}{c}\text { FMT Data } \\
\text { Distribution }\end{array}$ & Component & $\begin{array}{c}\text { Frequency } \\
\text { Diversity }\end{array}$ & $\begin{array}{c}\text { Time } \\
\text { Diversity }\end{array}$ \\
\hline $\mathbf{1}^{\text {st }}$ & Pilot & Max & Low \\
\hline $\mathbf{2}^{\text {nd }}$ & $\begin{array}{c}\text { Quasi-Pilot / Data } \\
\text { with geo-ranging }\end{array}$ & Max & Low \\
\hline $\mathbf{3}^{\text {rd }}$ & $\begin{array}{c}\text { Data with geo- } \\
\text { ranging }\end{array}$ & Max & High \\
\hline $\mathbf{4}^{\text {th }}$ & Data no geo-ranging & Max & High \\
\hline
\end{tabular}
\end{tabular}

TABLE IV. FMT CORRELATORS SUMMARY (1/2)

\begin{tabular}{|c|c|c|c|}
\hline $\begin{array}{c}\text { Correlator/ } \\
\text { Demodulator }\end{array}$ & $\begin{array}{c}\text { C/No } / N_{0} \text { at the } \\
\text { correlator output }\end{array}$ & $\begin{array}{c}\text { Improvement with } \\
\text { non-coherent } \\
\text { accumulation }\end{array}$ & Requirements \\
\hline A & Best & High & None \\
\hline B & Worst & Low & None \\
\hline C & $\begin{array}{c}\text { Depends on } \mathrm{L} . \\
\text { From worst }(\mathrm{L}=1) \\
\text { to best }\left(\mathrm{L}=\mathrm{L}_{\max }\right)\end{array}$ & $\begin{array}{c}\text { Depends on } \mathrm{L} . \\
\text { From low }(\mathrm{L}=1) \text { to } \\
\text { high }\left(\mathrm{L}=\mathrm{L}_{\max }\right)\end{array}$ & $\begin{array}{c}\text { Symbol group } \\
\text { starting point }\end{array}$ \\
\hline $\begin{array}{c}\text { Dem. } \mathbf{4}^{\text {th }} \\
\text { data distr. }\end{array}$ & The best one & -- & $\begin{array}{c}\text { Symbol group } \\
\text { starting point }\end{array}$ \\
\hline
\end{tabular}

TABLE V. FMT CORRELATORS SUMMARY $(2 / 2)$

\begin{tabular}{|c|c|c|c|}
\hline $\begin{array}{c}\text { Correlator/ } \\
\text { Demodulator }\end{array}$ & $\begin{array}{c}\text { Tracking } \\
\text { performance }\end{array}$ & $\begin{array}{c}\text { Data } \\
\text { Demodulation } \\
\text { Performance }\end{array}$ & $\begin{array}{c}\text { Acquisition } \\
\text { performance }\end{array}$ \\
\hline A & Best & $\begin{array}{c}\text { No time } \\
\text { diversity }\end{array}$ & High \\
\hline B & Worst & $\begin{array}{c}\text { High time } \\
\text { diversity }\end{array}$ & Low \\
\hline C & Best & $\begin{array}{c}\text { High time } \\
\text { diversity }\end{array}$ & $\begin{array}{c}\text { Depends on L. Search } \\
\text { range increase: a } \\
\text { mum of 5(L-1)M or } \\
\text { 7(L-1)M times the } \\
\text { original range }\end{array}$ \\
\hline $\begin{array}{c}\text { Dem. 4 } \\
\text { distr. }\end{array}$ & --- & $\begin{array}{c}\text { Highest time } \\
\text { diversity }\end{array}$ & --- \\
\hline
\end{tabular}

\section{CONCLUSIONS}

In this paper different data distributions have been presented for a FMT signal having each subcarrier broadcasting its own PRN code in time (MC-DS-CDMA type of signal). For each data distribution in the FMT signal a different correlator block suiting the data distribution purposes is presented. Moreover, the no use of FFT-based type of correlators has been justified and the mitigation of the Doppler Effect has been described. The conclusions about the different data distributions and their associated correlator blocks are given below.

Demodulation performance: When geo-ranging capabilities have to be provided, the $3^{\text {rd }}$ data distribution is a priori better than the $2^{\text {nd }}$ data distribution since it offers a better time diversity. When geo-ranging capabilities do not have to be provided, the best option is the $4^{\text {th }}$ data distribution since it provides the largest time diversity. 
Tracking performance: Since the symbol-synchronization is assumed to be achieved, from $1^{\text {st }}$ to $3^{\text {rd }}$ data distributions provide the same tracking performance.

Acquisition performance: The $1^{\text {st }}$ and $2^{\text {nd }}$ data distributions provide the best acquisition performance. The $3^{\text {rd }}$ data distribution should use first correlator B for a rough search and correlator B for the final precise search. The difference of acquisition performance between the correlators decreases along the signal $\mathrm{C} / \mathrm{N}_{0}$ increase.

To sum up, for only demodulation purposes the use of the $4^{\text {th }}$ data distribution is recommended. For geo-ranging purposes in addition to demodulation purposes, the system should find a data distribution structure between the $2^{\text {nd }}$ and $3^{\text {rd }}$ data distributions which provides the desired trade-off between demodulation and geo-ranging performances.

\section{ACKNOWLEDGMENT}

This paper is based upon the work done in the framework of the European Space Agency (ESA) funded study ADVISE, Advanced Signal-In-Space Techniques. The content of the present article reflects solely the authors' view and by no means represents the official ESA view or the one from ESA Galileo Project Office.

\section{REFERENCES}

[1] P.A. Dafesh and C.R. Cahn, "Phase-Optimized Constant-Envelope Transmission (POCET) Modulation Method for GNSS Signals" 22nd nternational Meeting of the Satellite Division of The Institute of Navigation, Savannah, GA, September 22-25, 2009

[2] L. Lestarquit and P-A. Brison, "Signal Choice for Galileo Compared Performances of Two Candidate Signals", Proceedings of the 2000 National Technical Meeting of The Institute of Navigation, Anaheim, CA January 26-28, 2000

[3] J. H. Won, M. Paonni, D. Fontanella, and B. Eissfeller, "Receiver Performance Analysis of Advanced Signal-In-Space Modulation Techniques for Next Generation GNSS", Proc. the 18th GNSS Workshop, Jeju, S. Korea, 3-4 Nov. 2011.

[4] A. Emmanuele, M. Luise, J. H. Won, D. Fontanella, M. Paonni, B. Eissfeller, F. Zanier and G. Lopez-Risueno, "Evaluation of Filtered Multitone (FMT) Technology for Future Satellite Navigation Use", Proc. ION GNSS-2011, Portland, OR, 20-23 Sept. 2011

[5] A.J. Van Dierendonck, "Global Positioning System: Theory and Applications", Progress in Astronautics and Aeonautics vol 164, 1996.

[6] S. Hara and R. Prasad, "Overview of Multicarrier CDMA", in Communications Magazine, IEEE, vol 35, page 126-133, Dec 1997.

[7] F. Bastide, O. Julien, C. Macabiau and B. Roturier, "Analysis of L5/E5 Acquisition, Tracking and Data Demodulation Thresholds", Proceedings of the 15th International Technical Meeting of the Satellite Division of The Institute of Navigation (ION GPS 2002), Portland, OR, September $24-27,2002$

[8] E. Kaplan and C. Hegarty, "Understanding GPS: Principles And Applications", Artech House, 2005. 